\title{
有机聚合物负载铑催化剂在氢甲酰化反应中的应用研究进展
}

\author{
宗玲博 $a$ 陈建宾 ${ }^{b}$ 任新意 $c$ 张国营 ${ }^{a}$ 贾肖飞*, $a$ \\ $(a$ 青岛科技大学化学与分子工程学院 山东省生化分析重点实验室 山东青岛 266042) \\ ( ${ }^{b}$ 齐鲁工业大学化学与制药工程学院 山东省分子工程重点实验室 济南 250353) \\ ( 华东师范大学化学与分子工程学院 绿色化学与化工过程绿色化上海市重点实验室 上海 200241)
}

\begin{abstract}
摘要 氢甲酰化反应是工业上最重要的均相反应之一. 该反应被广泛应用于醛的生产, 生产的醛还可以进一步转为具 有高附加值的醇、酸等衍生物. 与均相反应相比，多相催化在催化剂循环、产物和催化剂分离等方面具有优势. 近年来， 有机聚合物负载铑催化剂在非均相的甲酰化反应中表现出优秀的催化活性、高的选择性和好的循环性等优点, 因而受 到广泛关注. 总结了有机聚合物负载催化剂在氢甲酰化反应中应用研究进展, 主要介绍了催化剂的合成、材料特点和 应用，最后对发展前景进行了展望.
\end{abstract}

关键词 铑; 氢甲酰化; 有机聚合物; 非均相催化剂

\section{Progress in Application of Organic Polymers Supported Rhodium Catalysts in Hydroformylation}

\author{
Zong, Lingbo $^{a} \quad$ Chen, Jianbin $^{b} \quad$ Ren, Xinyi ${ }^{c} \quad$ Zhang, Guoying $^{a} \quad$ Jia, Xiaofei*, $^{*}$ \\ ( ${ }^{a}$ Shandong Key Laboratory of Biochemical Analysis, College of Chemistry and Molecular Engineering, \\ Qingdao University of Science and Technology, Qingdao, Shandong 266042) \\ $\left({ }^{b}\right.$ Shandong Provincial Key Laboratory of Molecular Engineering, School of Chemistry and Chemical Engineering, \\ Qilu University of Technology, Jinan 250353) \\ ( ${ }^{c}$ Shanghai Key Laboratory of Green Chemistry and Chemical Process, School of Chemistry and Molecular \\ Engineering, East China Normal University, Shanghai 200241)
}

\begin{abstract}
Hydroformylation is considered one of the most important homogenously catalyzed processes in dustry. Hydroformylation has been widely used in the production of aldehydes, and aldehydes can also be further converted into high value-added alcohols, acids and other derivatives. Compared with the homogeneous reaction, the heterogeneous catalysts present significant advantages in terms of recyclability, separation of catalysts and products and so on. In recent years, organic polymer-supported rhodium catalysts have shown excellent catalytic activity, high selectivity, and good recycleability in heterogeneous hydroformylation, and have attracted widespread attention. The research progress of the application of organic polymer supported catalysts in hydroformylation is summarized, including synthesis, material characteristics and application of supported catalysts. Finally, the prospect of the reaction is discussed.
\end{abstract}

Keywords rhodium; hydroformylation; organic polymers; heterogeneous catalysts

氢甲酰化反应也称之为 “Oxo 合成”, 是指在催化 剂的作用于下, 烯烃与 $\mathrm{H}_{2} / \mathrm{CO}$ 反应得到醛的过程 ${ }^{[1]}$, 1938 年由德国科学家 Roellen 首次发现 ${ }^{[2]}$. 经过几十年 的发展, 氢甲酰化反应作为工业中最重要的均相反应之 一, 已经是商业上合成醛主要的方法, 每年生成的醛及 其衍生物达一千万多吨 ${ }^{[3]}$. 醛作为重要的化工原料, 不
仅可以用作香料的添加剂, 而且经氢化、氧化、羟醛缩 合、还原胺化等反应制备醇、酸、 $\alpha, \beta$-不饱和醛酮和胺 等化合物(Scheme 1). 另外, 氢甲酰化反应是一类 100\% 原子经济性制备醛的过程. 因此, 研究氢甲酰化反应具 有重要的理论意义和应用价值.

\footnotetext{
* Corresponding author. E-mail: jiaxiaofei139@163.com

Received March 3, 2020; revised May 1, 2020; published online May 19, 2020.

Project supported by the National Natural Science Foundation of China (Nos. 21703116, 51702180) and the Open Fund of the Department of Chemistry, Qingdao University of Science and Technology (Nos. QUSTHX201932, QUSTHX202010).

国家自然科学基金(Nos. 21703116, 51702180)、青岛科技大学化学学部开放基金(Nos. QUSTHX201932, QUSTHX202010)资助项目.
} 


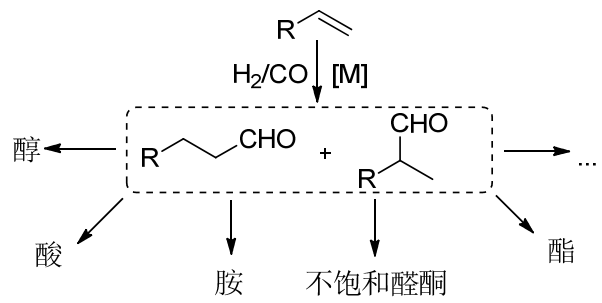

图式 1 氢甲酰化产物醛及衍生物

Scheme 1 Hydroformylation of olefins to aldehydes and their derivatives

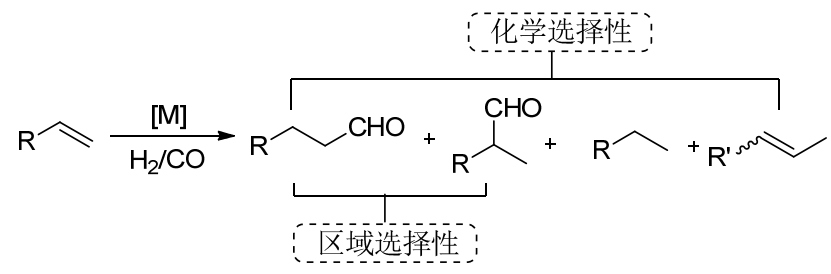

图式 2 氢甲酰化反应中的选择性

Scheme 2 Selectivity in hydroformylation

在取代烯烃的氢甲酰化反应过程中，不仅存在着反 应活性问题, 而且还有区域选择性和化学选择性问题 (Scheme 2). 醛基可以生成在双键的末端和内端, 分别 得到直链醛和支链醛. 目前, 直链醛是工业上的主要需 求, 如直链醛衍生的直链醇广泛应用于洗涤剂和增塑剂 的原材料. 另外, 在反应中还伴随着氢化产物和异构化
烯烃的生成. 催化剂对氢甲酰化反应的活性和选择性起 着决定性作用. 因此, 发展高活性和高选择性的催化剂 具有重要的意义. 钴是最早应用于工业氢甲酰化反应的 催化剂. 由于存在着催化活性低、高温高压及化学选择 性差等问题，工业上钴催化剂逐渐被铑催化剂代替. 在 铑催化的氢甲酰化反应中，配体对反应的活性、区域选 择性和化学选择性具有重要的决定性作用. 一系列的膦 配体被用于均相铑催化的烯烃氢甲酰化反应，代表性的

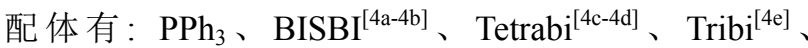
Naphos $^{[5]} 、 X^{2}$ antphos ${ }^{[6]}$ 、亚磷酸酯配体(DSM ligand ${ }^{[7]}$, Biphephos ${ }^{[8]}$ )和吡咯基亚磷酰胺配体配体 ${ }^{[9]}$ 等 (Scheme 3).

与均相反应相比, 非均相催化体系具有产物与催化 剂分离容易，催化剂可回收循环利用等优点，更具有广 阔的工业应用前景. 在非均相氢甲酰化反应中，首先需 要解决几个主要问题: (1)达到与均相体系相当的或更高 的催化剂活性; (2)反应的选择性, 包括直链醛和支链醛 的选择性及异构化产物和氢化产物; (3)催化剂的流失, 包括配体和铑金属的流失; (4)催化剂的循环次数及使用 寿命.

实现均相氢甲酰化反应的非均相化，主要包括液/ 液两相和液/固两相催化体系 ${ }^{[10]}$. 液/液两相催化体系分<smiles>c1ccc(P(c2ccccc2)c2ccccc2)cc1</smiles>

$\mathrm{PPh}_{3}$<smiles>c1ccc(P(Cc2ccccc2-c2ccccc2P(c2ccccc2)c2ccccc2)c2ccccc2)cc1</smiles>

$\mathrm{BISBI}$<smiles>Pc1cccc(Cc2ccccc2)c1-c1c(Cc2ccccc2Cc2ccccc2)cccc1Pc1ccccc1</smiles><smiles>c1ccc(Cc2cccc(Cc3ccccc3)c2-c2ccccc2)cc1</smiles><smiles>COc1cc(-c2cc(OC)cc(C(C)(C)C)c2Op2oc3ccccc3c3ccccc3o2)c(Op2oc3ccccc3c3ccccc3o2)c(C(C)(C)C)c1</smiles>

Biphephos

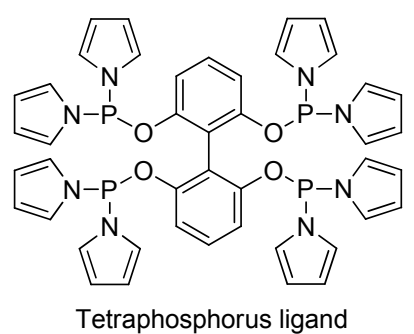

Tetraphosphorus ligand<smiles>c1ccc(P(Cc2ccc3ccccc3c2-c2c(P(c3ccccc3)c3ccccc3)ccc3ccccc23)c2ccccc2)cc1</smiles>

Naphos<smiles>CC1(C)c2cccc(P(c3ccccc3)c3ccccc3)c2Oc2c(P(c3ccccc3)c3ccccc3)cccc21</smiles>

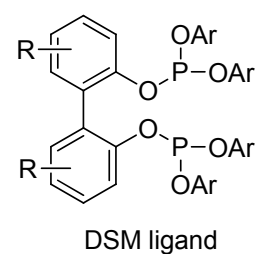<smiles></smiles><smiles>c1ccc(P(n2cccc2)n2cccc2-c2ccccc2-c2c(OP(n3cccc3)n3cccc3)cccc2OP(n2cccc2)n2cccc2)cc1</smiles>

图式 3 氢甲酰化反应中的代表性膦配体

Scheme 3 Representative phosphine ligands in the hydroformylation 
为水/有机两相 ${ }^{[11]}$ 、氟两相 ${ }^{[12]}$ 、离子液体两相 ${ }^{[13]}$ 、超临 界流体 ${ }^{[14]}$ 等. 但用于工业化只有水/有机两相催化体系, 代表性的为 $\mathrm{RCH} / \mathrm{RP}$ 工艺 ${ }^{[15]}$. 水/有机两相是指以水为 反应溶剂, 使用水溶性的磺酸钠膦配体与铑络合催化烯 烃的氢甲酰化反应; 反应之后产物醛和水溶液为两相, 且催化剂溶于水相, 所以容易实现产物和催化剂的分离 及回收. 目前用于水/有机两相的膦配体包括 TPPTS (trisulfonated triphenylphosphane) ${ }^{[16]}$, BISBIS (sulfonated 2,2'-bis(diphenylphosphinomethyl)-1,l'-biphenyl) ${ }^{[17]}$ 和 BINAS (bis[disulphonatophenylphosphinomethyltetrasulphonatobinaphthene] $)^{[18]}$ (Scheme 4). 由于烯烃在水相中 的溶解性问题, 该工艺主要用于短链 $\mathrm{C}_{3}$ 和 $\mathrm{C}_{4}$ 烯烃的氢 甲酰化反应.

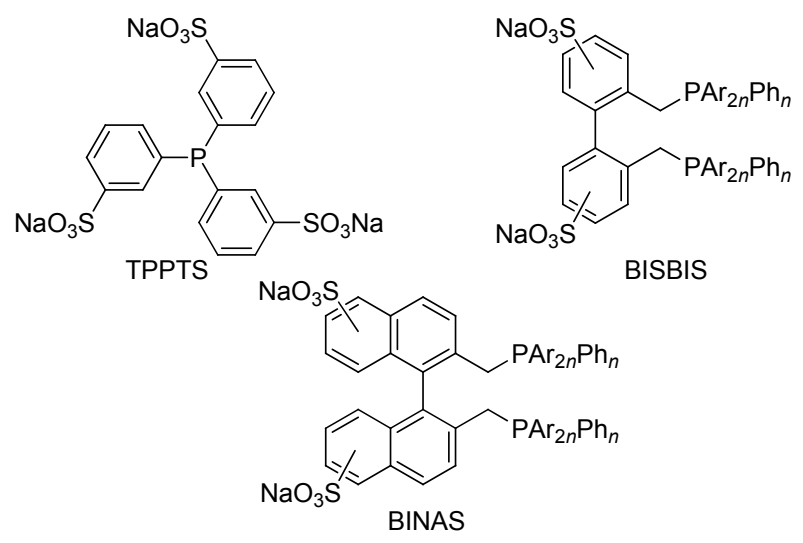

图式 4 用于水/有机两相的氢甲酰化反应的膦配体

Scheme 4 Phosphine ligands used for aqueous two-phase hydroformylation

液/固两相体系是指将催化剂负载于固体载体上, 产物 醛为液相, 反应完成后容易实现产物与催化剂的分离. 固载载体分为无机载体和聚合物载体两大类. 无机载体 主要包括分子篮 ${ }^{[19]}$ 、碳材料 ${ }^{[20]}$ 和金属氧化物 ${ }^{[21]}$ 等. 通过 利用无机材料的多孔结构, 包覆、封装或共价键结合固 载催化剂. 该类固载催化剂可以有效解决催化剂的活 性, 但并不能得到理想的区域选择性. 氢甲酰化反应的
催化剂中，配体对铑金属的反应活性尤其是选择性起到 了主要的决定性作用. 由于无机载体本身的局限性，使 得其与有机膦配体之间难以构建稳定的键合结构，导致 无法满足固载催化剂中铑周围的配体浓度，而且在反应 中容易发生膦配体的流失. 低的配体浓度及配体流失将 会引起反应选择性的降低和铑金属的流失，进而影响催 化剂的使用寿命. 另外, 使用无机载体难以实现配体修 饰的多样化.

以有机聚合物为载体，将膦配体通过共价键固定在 聚合物链中，进一步通过膦配体与铑金属的配位实现催 化剂的负载. 聚合物固载催化剂可以实现负载膦配体的 多样化, 有效提高催化剂性能和稳定性. 因此, 本文概 述了近年来有机聚合物负载催化剂在氢甲酰化反应中 应用研究进展, 探讨了固载催化剂的合成、结构与氢甲 酰化反应活性、选择性和稳定性之间的关系.

\section{1 传统有机聚合物负载催化剂}

传统的有机聚合物主要包括聚苯乙烯、聚甘油和壳 聚糖等. 经聚合反应和缩合反应等, 以 $\mathrm{C}-\mathrm{C} 、 \mathrm{C}-\mathrm{O} 、 \mathrm{C}$ 一 $\mathrm{N}$ 等共价键等将配体固定在聚合物链中. 在 1998 年, Nozaki 课题组 ${ }^{[22]}$ 用乙烯基修饰的 $(R, S)$-BINAPHOS $((R)-(2-(d i p h e n y l p h o s p h i n o)-1,1 '-b i n a p h t h a l e n-2 '-y l)-((S)-1$ ,l'-binaphthalen-2,2'-yl)phosphite)先与 $\mathrm{Rh}(\mathrm{acac})(\mathrm{CO})_{2}$ 络 合，再将其与商业化的二乙烯基苯聚合可制备得到 PS-[Rh(acac) $(R, S)-B I N A P H O S]$ (Scheme 5). 该方法制 备的催化剂在苯乙烯和醋酸乙烯酯的不对称氢甲酰 化反应中均可以取得与均相体系相当的催化效果 (Schemes 6a, 6b). 另外, 该类催化剂不会在反应中发生 溶胀, 容易实现催化剂的分离. 2003, Nozaki 课题组 ${ }^{[23]}$ 使用固定床反应器探索了 PS-[Rh(acac) $(R, S)$-BINAPHOS]催化剂在氢甲酰化反应中的使用时间和循环次 数. 当以液化的顺式-二丁烯为反应底物和反应溶剂时, 反应 $48 \mathrm{~h}$ 后, 产物醛的 $e e$ 值由最初的 $79 \%(12 \mathrm{~h})$ 降至

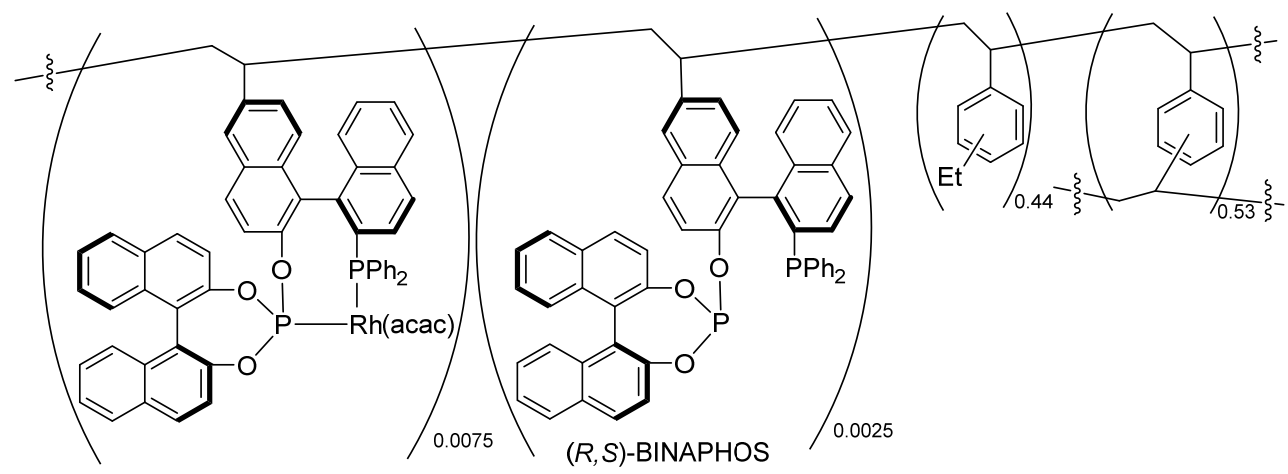

图式 5 聚苯乙烯负载的 $(R, S)$-BINAPHOS-Rh(I)

Scheme 5 Polystyrene-supported $(R, S)$-BINAPHOS-Rh(I) 
(a)

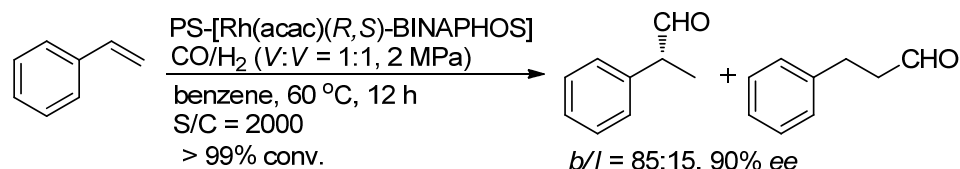
$>99 \%$ conv. $b / l=85: 15,90 \%$ ee

(b) $\mathrm{AcO} \curvearrowright$ $\mathrm{PS}-[\mathrm{Rh}(\mathrm{acac})(R, S)-\mathrm{BINAPHOS}]$ $\underset{\text { benzene, } 60^{\circ} \mathrm{C}, 42 \mathrm{~h}}{\mathrm{CO}}$ $\mathrm{S} / \mathrm{C}=500$ $67 \%$ conv.

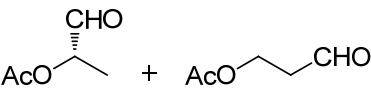
$b / I=87: 13,92 \%$ ee $\mathrm{PS}-[\mathrm{Rh}(\mathrm{acac})(R, S)-\mathrm{BINAPHOS}]$

(c)

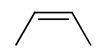
$\mathrm{CO} / \mathrm{H}_{2}(V: V=1: 1,3.3 \mathrm{MPa})$ $60^{\circ} \mathrm{C}, t=12 \sim 48 \mathrm{~h}$ TOF $=12 \sim 76 \mathrm{~h}^{-1}$ TON $=156 \sim 3264$

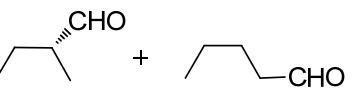
$b / I=100: 0 \sim 92: 8,79 \% \sim 7 \%$ ee

(d) $\mathrm{F}_{3} \mathrm{\curvearrowright}$ PS-[Rh(acac)(R,S)-BINAPHOS] total pressure $5 \mathrm{MPa}$ $60^{\circ} \mathrm{C}, t=0.5 \mathrm{~h}$ TOF $=9 \mathrm{~h}^{-1}$ $\mathrm{TON}=4.5$<smiles>CC([CH+]C(CO)C(F)(F)F)C(F)(F)CCCO</smiles>
PS-[Rh(acac) $(R, S)-B I N A P H O S]$

(e)<smiles>C=Cc1ccccc1</smiles>
$\mathrm{CO} / \mathrm{H}_{2}(V: V=1: 1,12 \mathrm{MPa})$ $60^{\circ} \mathrm{C}$ conv. $81 \% \sim 94 \%$ 6 cycles $b / l=95: 5,90 \%$ ee

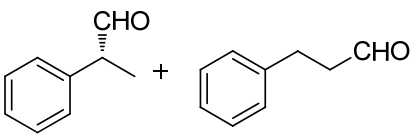

iso-aldehyde $76 \% \sim 80.3 \%$, $82 \% \sim 86 \%$ ee

图式 6 PS-[Rh(acac) $(R, S)$-BINAPHOS]催化的烯烃的不对称氢甲酰化反应

Scheme 6 PS-[Rh(acac) $(R, S)$-BINAPHOS] catalyzed asymmetric hydroformylation of olefins

$7 \%$ (Scheme 6c). 当以三氟甲基乙烯为反应底物时，产 物的对映选择性可以保持, 但催化剂的活性明显降低 (Scheme 6d). 为了提高催化剂的活性, 当采用超临界 $\mathrm{CO}_{2}$ 作为反应介质和高的合成气压力 $(12.1 \mathrm{MPa})$ 条件下, 催化剂能够循环使用 6 次, 且保持好的催化活性和选择 性(Scheme 6e)

树枝状聚甘油是一类含有大量羟基的超支化聚合 物, 通过共价键的形式将催化剂固定在其分子内部或周 围，来提高催化剂稳定性、分散性和催化效率 ${ }^{[24]} 2006$ 年, Haag 和 Eilbracht 课题组 ${ }^{[25]}$ 分别通过迈克尔加成反 应和异凗酸酯的亲核加成反应, 将修饰的 NIXANTPHOS [4,6-bis(diphenylphosphino)-10H-phenoxazine] 固 定在树状的聚甘油链中, 进一步通过与铑催化前体络合 分别得到两类聚合物负载催化剂 PG-NIXAN-TPHOS$\mathrm{Rh}(\mathrm{I})$ 和 PG/PU-NIXANTPHOS-Rh(I) (Scheme 7). 在 PG-NIXANTPHOS-Rh(I)催化的 $N$-烯丙基邻苯二甲酰亚 胺的氢甲酰化反应中, 仅以 $30 \% \sim 40 \%$ 的转化率和低的 区域选择性 $(l: b=38: 62)$ 得到相应脂肪醛(Scheme $8 \mathrm{a})$. 在 PG/PU-NIXANTPHOS-Rh(I)催化的 1-辛烯的氢 甲酰化反应中, 以 $69 \%$ 的转化率和高的区域选择性 $(l$ : $b=96.8: 3.2$ ) 得到相应的醛产物(Scheme $8 \mathrm{~b}$ ). 但这两 类负载催化剂的催化性能均低于相应的均相催化体系.

壳聚糖是甲壳素脱乙酰化的产物, 具有无毒无害易 降解的特点. 在壳聚糖的分子链中含有大量的氨基和羟 基，易于通过化学修饰来负载催化剂 ${ }^{[26]} .2012$ 年, Smith
课题组 ${ }^{[27]}$ 以壳聚糖和 2 -苯基膦苯甲醛为原料, 经缩合反 应得到含有 Schiff 碱的壳聚糖. 通过壳聚糖中 $\mathrm{C}=\mathrm{N}$ 和 $\mathrm{PPh}_{2}$ 单元与铑的配位实现催化剂的固载(Scheme 9a). 该类催化剂在 1-辛烯的氢甲酰化反应中，能够循环使用 4 次，以 $75 \% \sim 79 \%$ 的转化率和中等的区域选择性 $(l$ :

(a)

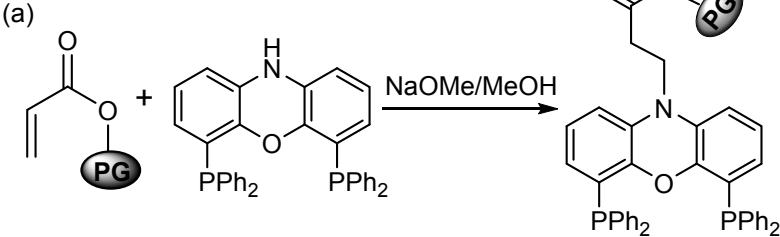

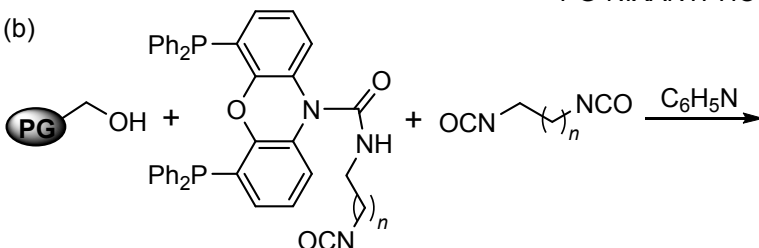

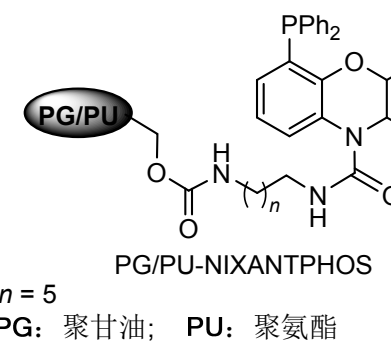

图式 7 PG-NIXANTPHOS 和 PG/PU-NIXANTPHOS 的合成 Scheme 7 Synthesis of PG-NIXANTPHOS and PG/PU-NIXANTPHOS 

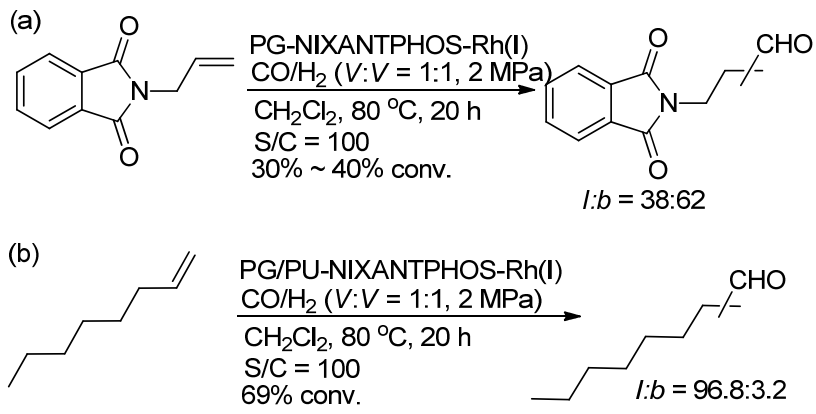

图式 8 PG-NIXANTPHOS-Rh(I)和 PG/PU-NIXANTPHO $S$ $\mathrm{Rh}(\mathrm{I})$ 催化的烯烃氢甲酰化反应

Scheme 8 PG-NIXANTPHOS-Rh(I) and PG/PU-NIXANT PHOS-Rh(I) catalyzed hydroformylation of olefins

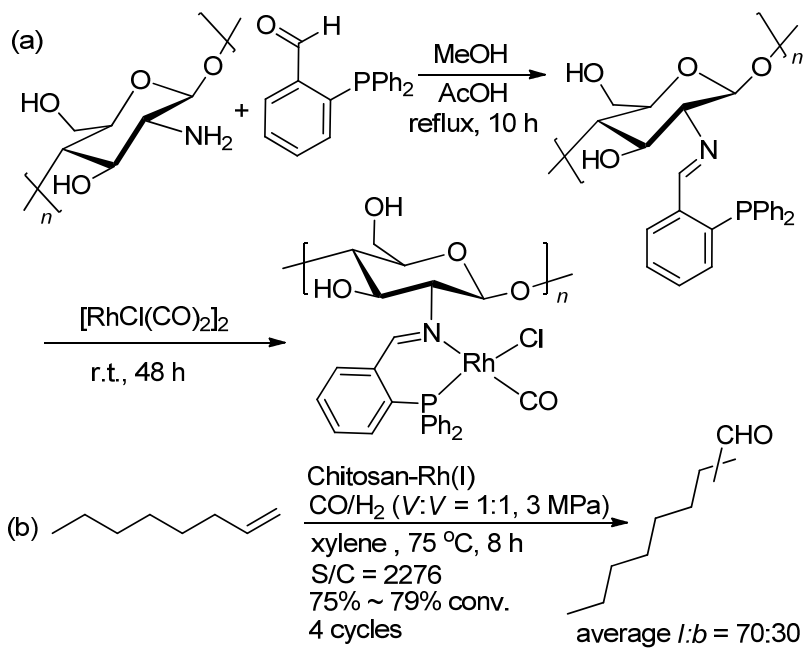

图式 9 壳聚糖负载铑催化剂的制备及应用

Scheme 9 Synthesis and application of chitosan-supported Rh catalyst

$b=70 ： 30$ )得到相应的醛 (Scheme 9b). 在滤液中, 经 ICP-MS 可以检测到 $0.02 \%$ 的铑的流失.

从以上研究结果可以发现, 聚合物可以实现催化剂 修饰的的多样性, 但传统的有机聚合物的比表面积比较 低, 导致有些固载催化剂难以达到相应的均相催化剂活 性. 另外, 由于配体是固定在其它的有机聚合物链中, 并不能控制配体的分布及铑金属周围的配体浓度，因而 反应的选择性也会受到一定的影响，同时导致铑的流 失, 进而影响催化剂的循环.

\section{2 多孔有机聚合物负载催化剂}

近年来, 多孔有机聚合物(POPs)的发展成为一个新 的研究热点 ${ }^{[28]}$. 该类材料可以应用于气体吸收、分子分 离和电化学等方向, 同时也为负载催化剂提供了一类新 的聚合物载体. 尤其是以有机配体单体聚合构建的多孔 有机聚合物(POLs)作为负载体具有以下优势: (1)相对比 传统的聚合物载体, 多孔配体聚合物具有可更高的比表 面积, 可以有效分散金属催化剂, 防止催化剂的聚集.
另外，由于该类聚合物为交联产物，所以不溶于大多数 溶剂，防止溶解而导致的催化剂损失. (2)该类聚合物具 有多孔结构. 有利于反应物与催化活性位点的接触及产 物的扩散. 同时, 根据反应需要, 可以调节孔径的大小. (3)该类聚合物负载金属催化剂时，主要通过聚合物中 的配体单元与金属配位螯合，这样容易形成单原子催化 中心，使得催化剂具有更高的催化活性. (4)在负载的金 属中心周围有很多的配体单元，高浓度的配体可以稳定 催化剂，防止金属的流失，使催化剂具有更多的循环次 数和使用时间. 基于以上的特点, 该类多孔聚合物负载 铑催化剂在氢甲酰化的反应中表现出优异的催化性能.

\section{1 单齿膦配体均聚物负载催化剂}

均聚物负载催化剂是指由一种单体聚合而成的聚 合物负载的催化剂. 构建多孔结构聚合物往往需要反应 单体包含两个或两个以上的聚合反应位点. 因此, 从合 成的角度出发, 就需要对膦配体进行进一步的修饰. 结 合目前的研究工作，聚合单元以乙烯基基团为主. 在膦 配体结构中构建乙烯基单元主要包括三种方法: (1)直接 使用乙烯基的原料; (2)使用含有羰基的原料，通过 Wittig 反应合成乙烯基; (3)通过在配体骨架上引入卤素, 通过偶联反应合成乙烯基.

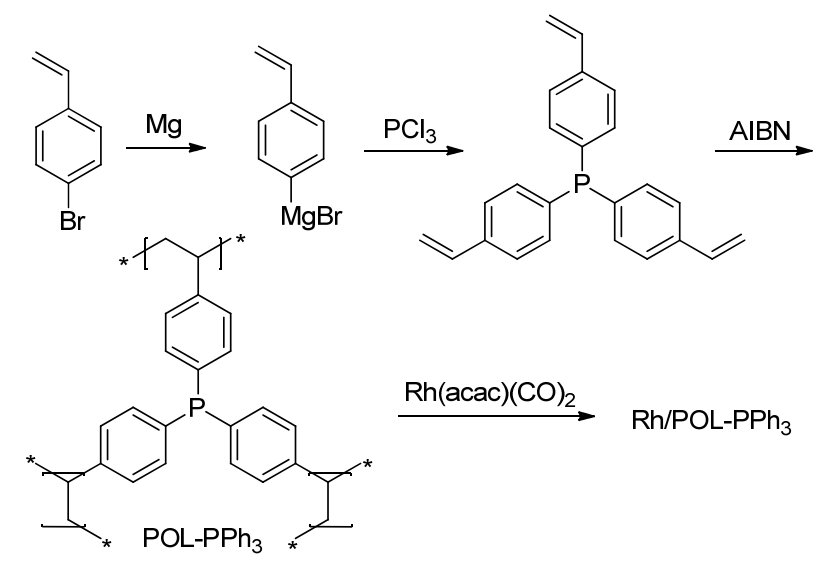

图式 $10 \mathrm{Rh}(\mathrm{CO})_{2}(\mathrm{acac}) / \mathrm{POL}-\mathrm{PPh}_{3}$ 催化剂的合成 Scheme 10 Synthesis of $\mathrm{Rh}(\mathrm{CO})_{2}(\mathrm{acac}) / \mathrm{POL}-\mathrm{PPh}_{3}$

2014 年, 丁云杰课题组和刘景月课题组 ${ }^{[29]}$ 报道了 $\mathrm{Rh}(\mathrm{CO})_{2}(\mathrm{acac}) / \mathrm{POL}-\mathrm{PPh}_{3}$ 催化剂的合成，并将其应用于 1-辛烯的氢甲酰化反应. 以对溴苯乙烯为起始原料制备 格氏试剂，再与 $\mathrm{PCl}_{3}$ 反应得到三乙烯基三苯基膦; 在 AIBN 的催化下，乙烯基三苯基膦单体经过聚合反应得 到 $\mathrm{POL}-\mathrm{PPh}_{3}(\mathrm{Scheme} 10)$. 经结构表征, 聚合物

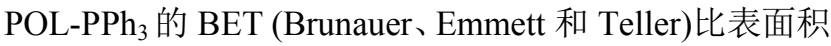
和孔容分别可达到 $1086 \mathrm{~m}^{2} \cdot \mathrm{g}^{-1}$ 和 $1.70 \mathrm{~cm}^{3} \cdot \mathrm{g}^{-1}$; 孔径主 要分布在 $0.7 、 1.5$ 和 3 70 nm, 说明该类材料含有多级 孔状结构(表 1 , Entry 1); 分解温度可以高达 $440{ }^{\circ} \mathrm{C}$, 甚 
表 1 多孔聚合物和及其负载催化剂的结构参数

Table 1 Textural parameters of porous organic polymers and their supported catalysts

\begin{tabular}{|c|c|c|c|c|c|c|}
\hline Entry & Sample & $\begin{array}{l}\text { BET surface } \\
\text { area } /\left(\mathrm{m}^{2} \cdot \mathrm{g}^{-1}\right)\end{array}$ & $\begin{array}{l}\text { Pore volume/ } \\
\left(\mathrm{cm}^{3} \cdot \mathrm{g}^{-1}\right)\end{array}$ & $\begin{array}{l}\text { Pore size distribution/ } \\
\mathrm{nm}\end{array}$ & $\begin{array}{l}\text { Decomposition } \\
\text { temperature } /{ }^{\circ} \mathrm{C}\end{array}$ & Reference \\
\hline 1 & POL-PPh 3 & 1086 & 1.70 & $0.7,1.5,3 \sim 70$ & 440 & [29] \\
\hline 2 & $\mathrm{Rh} / \mathrm{POL}_{-} \mathrm{PPh}_{3}$ & 1032 & 1.69 & - & - & [29] \\
\hline 3 & Phosphite-POP & 643 & 0.43 & $0.5-1.4,2 \sim 10$ & - & [31] \\
\hline 4 & $\mathrm{Rh} /$ phosphite-POP & 616 & 0.43 & - & - & [31] \\
\hline 5 & POL-dppe & 943 & 1.51 & $0.7-1.5,2.8 \sim 150$ & $>400$ & [33] \\
\hline 6 & $\mathrm{Rh} /$ POL-dppe & 856 & 1.41 & - & - & [33] \\
\hline 7 & POL-dppm & 892 & 1.07 & - & - & [33] \\
\hline 8 & POL-dppb & 846 & 0.81 & - & - & {$[33]$} \\
\hline 9 & POL-Xantphos\& $\mathrm{PPh}_{3}$ & 1022 & 1.24 & $0.6-1,1.2 \sim 2,2 \sim 10$ & $>450$ & [34] \\
\hline 10 & $\mathrm{Rh} / \mathrm{POL}-\mathrm{X} a n t p h o s \& \mathrm{PPh}_{3}$ & 883.9 & 1.16 & - & - & [34] \\
\hline 11 & CPOL-BP\&PPh & 1088.0 & 2.07 & $0.70,0.84,1.38,2.18$ & $>430$ & {$[35]$} \\
\hline 12 & $\mathrm{Rh} / \mathrm{CPOL}-\mathrm{BP} \& \mathrm{PPh}_{3}$ & 985.3 & 1.94 & - & - & {$[35]$} \\
\hline 13 & $\mathrm{Rh} / \mathrm{CPOL}-\mathrm{BP} \& \mathrm{Ph}$ & 1116 & 1.66 & - & - & {$[36]$} \\
\hline 14 & CPOL-BP\&P(OPh $)_{3}$ & 635 & 0.72 & $0.7,0.85,1.38,1.89,2 \sim 10$ & - & [37] \\
\hline 15 & $\mathrm{Rh} / \mathrm{CPOL}-\mathrm{BP} \& \mathrm{P}(\mathrm{OPh})_{3}$ & 556 & 0.68 & - & - & [37] \\
\hline 16 & $\mathrm{Rh} / \mathrm{CPOL}-\mathrm{BPa} \& \mathrm{PPh}_{3}$ & 423.6 & 0.41 & $1.2 \sim 2.0,2.3 \sim 25$ & 370 & {$[38]$} \\
\hline 17 & $\mathrm{Rh} / \mathrm{POL}-\mathrm{BINAPa} \& \mathrm{PPh}_{3}$ & 492.2 & 0.41 & - & 456 & {$[40]$} \\
\hline 18 & $\mathrm{PSA}^{-\mathrm{PPh}_{3}}$ & 547 & 0.56 & - & 400 & {$[41]$} \\
\hline 19 & PSA-Xantphos & 290 & 0.69 & - & - & [41] \\
\hline
\end{tabular}

至高于全氟磺酸树脂 Nafion NR50 的分解温度 $\left(330{ }^{\circ} \mathrm{C}\right)$, 表明 $\mathrm{POL}-\mathrm{PPh}_{3}$ 有好的热稳定性. 负载 $\mathrm{Rh}$ 以后的催化剂 仍能保持高的比表面积和孔容(表 1, Entry 2). 在 1/6000 的催化剂用量下, $\mathrm{Rh} / \mathrm{POL}-\mathrm{PPh}_{3}$ 催化的 1-辛烯的氢甲酰 化反应中, 能以 $88.1 \% \sim 89.7 \%$ 的收率和 $0.87 \sim 1.35$ 的正 异比得到目标醛; 催化剂循环使用 6 次，且保持稳定的 转化率、收率和选择性; 与均相的 $\mathrm{Rh} / \mathrm{PPh}_{3}$ 催化效果相 比, Rh/POL-PPh 3 能得到更高的区域选择性(Scheme 11).

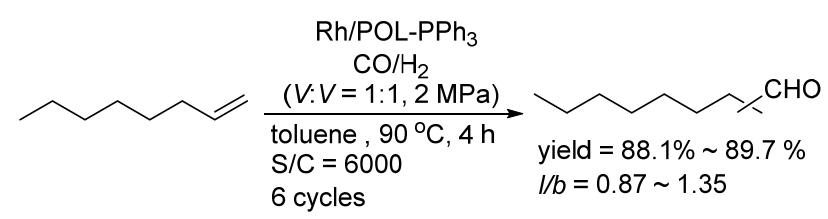

图式 $11 \mathrm{Rh} / \mathrm{POL}-\mathrm{PPh}_{3}$ 催化的烯烃氢甲酰化反应

Scheme $11 \mathrm{Rh} / \mathrm{POL}-\mathrm{PPh}_{3}$ catalyzed hydroformylation of olefins

2015 年, 丁云杰课题组和刘景月课题组 ${ }^{[30]}$ 研究了 $\mathrm{Rh} / \mathrm{POL}-\mathrm{PPh}_{3}$ 催化剂在固定床反应器中的氢甲酰化催化 性能. 当乙烯为反应底物时, 能以高的转化率 $(96.2 \%)$ 和 优秀的催化活性 $\left(\mathrm{TOF}=4530 \mathrm{~h}^{-1}\right.$ )得到丙醛产物; 催化 剂可以使用 1000 多个小时, 催化活性几乎没有降低且 在产物中未检测到铑的流失. 当长链的十二烯用于反应 时, 经过 $504 \mathrm{~h}$ 的反应, 转化率仍然保持在 $86.6 \%$, 且直 链醛与支链醛比值可以达到 6.4. 该结果进一步证明了 $\mathrm{Rh} / \mathrm{POL}-\mathrm{PPh}_{3}$ 催化性能和稳定性. 高角环形暗场扫描透 射电子显微镜(HAADF-STEM)显示, Rh/POL-PPh 催化 剂中 $\mathrm{Rh}$ 以单原子形态存在. 使用后的催化剂也没有出
现铑金属的烧结现象. 拓展 X 射线吸收精细结构谱图 (EXAFS)分析表明，在使用后的催化剂中含有 $\mathrm{Rh}-\mathrm{P}$ 和 $\mathrm{Rh}-\mathrm{C}$ 的键，并没有发现 $\mathrm{Rh}-\mathrm{Rh}$ 键，进一步说明了铑 的单原子形态，同时证明聚合物中 $\mathrm{Rh}$ 与 $\mathrm{PPh}_{3}$ 配位非常 稳定.

亚磷酸酯配体对水比较敏感，容易发生配体分解， 导致催化剂的活性和选择性的降低. 2016 年，肖丰收课 题组和马胜前课题组 ${ }^{[31]}$ 发展的亚磷酸酯均聚物, 在实 现催化剂固载化的同时，解决了亚磷酸酯对水的敏感问 题，代表性的聚合物为 Phosphite-POP. 以邻叔丁基苯酚 为底物, 经过六步反应得到乙烯基亚磷酸酯单体; 在偶 氮二异丁腈(AIBN)的引发下，单体经自由基反应得到 Phosphite-POP (Scheme 12). 聚合物 Phosphite-POP 的 BET 比表面积和孔容分别为 $643 \mathrm{~m}^{2} \cdot \mathrm{g}^{-1}$ 和 $0.43 \mathrm{~cm}^{3} \cdot \mathrm{g}^{-1}$; 孔径主要分布在 $0.5 \sim 1.4$ 和 $2 \sim 10 \mathrm{~nm}$, 表明聚合物由微 孔和介孔构成(表 1, Entry 3). 更重要的是, Phosphite-POP 表现出优异的超疏水性能; 该疏水性能能够 有效阻止水分子进入聚合物的孔隙，使得 Phosphite-POP 具有很好的水稳定性. 该聚合物负载 $\mathrm{Rh}(\mathrm{CO})_{2}$ (acac)前体得到 Rh/phos- phite-POP 催化剂. 在 具有挑战性的 2-辛烯的异构化/氢甲酰化反应中, Rh/phosphite-POP 表现出优秀的催化活性、化学选择性 和循序性，但反应的区域选择性偏低(Scheme 12).

之后, 肖丰收课题组又考察了 $\mathrm{Rh} /$ phosphite-POP 催 化剂中 $\mathrm{P} / \mathrm{Rh}$ 物质的量比 $(\mathrm{P}: \mathrm{Rh}=2,4,9,18,36)$ 对 2-辛 烯的异构化/氢甲酰化反应的催化活性、选择性及催化剂 


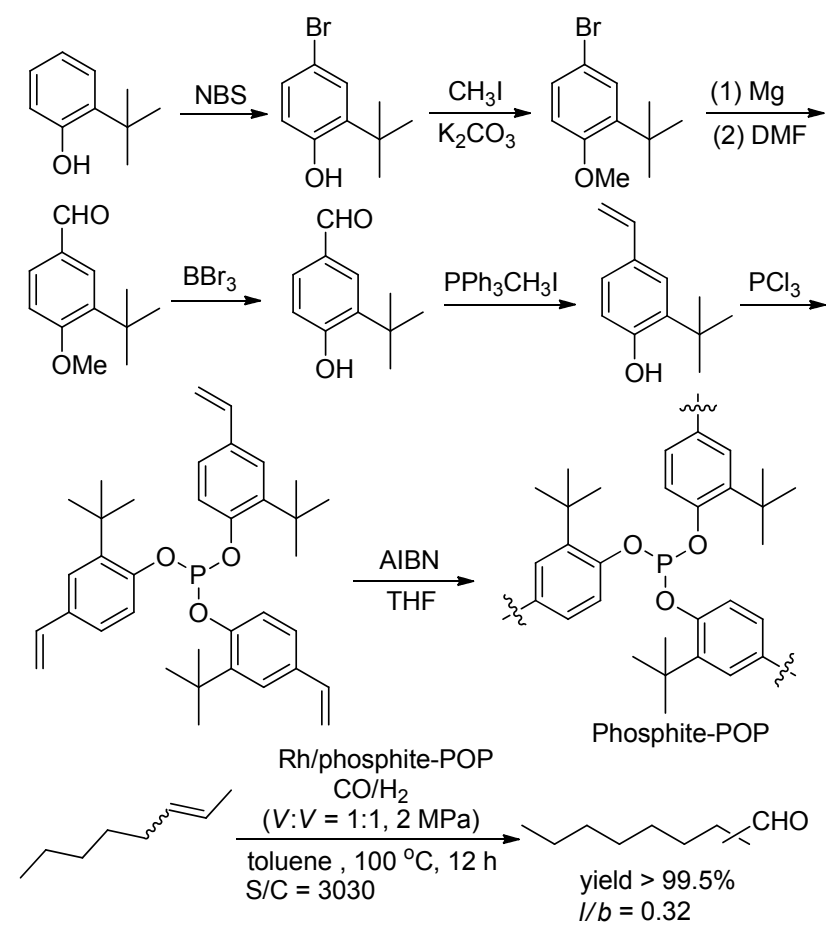

图式 $12 \mathrm{Rh} / \mathrm{phosphite-POP}$ 的合成和应用

Scheme 12 Synthesis and application of $\mathrm{Rh} /$ phosphite-POP

的循环性的影响 ${ }^{[32]}$. 在 $\mathrm{P} / \mathrm{Rh}$ 比为 9 时, 催化剂表现出最 高的反应活性, 降低和升高 $\mathrm{P} / \mathrm{Rh}$ 比都会引起催化活性 的降低; $\mathrm{P} / \mathrm{Rh}$ 比的改变并没有引起反应的化学选择性和 区域选择性的变化. 当固载催化剂的 $\mathrm{P} / \mathrm{Rh}$ 比大于 9 时, 催化剂循环十次仍然保持反应的活性和选择性, 经 ICP-OES 检测后, 未发现铑物种的流失; 当 $\mathrm{P} / \mathrm{Rh}$ 比小于 9 时 $(\mathrm{P}: \mathrm{Rh}=2,4)$, 五次循环之后, 铑的流失量分别达 到 $16.9 \%$ 和 $6.8 \%$, 且醛的收率逐渐降低. 由此可见, 膦 配体的浓度对催化剂的活性和铑的流失具有重要的决 定作用. 高浓度的膦配体可以稳定催化剂, 且自由的膦 配体可以配位流失的铑物种, 从而减少铑流失和保持催 化剂的活性. 另外, 以水作为反应溶剂, Rh/phosphitePOP 催化不同种类烯烃的氢甲酰化反应, 并以优秀的收 率得到目标醛(Scheme 13).

\section{2 双齿膦配体均聚物负载催化剂}

与单膦配体相比, 双膦配体与铑配体形成的络合物 具有更稳定的构型和空间立体效应, 用于催化烯烃氢甲 酰化反应时往往可以取得更高的选择性. 2015 年, 肖丰 收课题组 ${ }^{[33]}$ 发展了一系列双齿膦配体聚合物负载铑催 化剂, 并将其用于烯烃的氢甲酰化反应研究. 用 4-乙烯 苯基格氏试剂与不同的烷基膦氯和芳基膦氯反应, 分别 得到不同的乙烯基双膦配体单体 dppm [bis-(diphenylphosphino)methane] 、 dppe [1,2-bis(diphenylphosphino)ethane] 和 $\mathrm{dppb}$ [1,4-bis(diphenylphosphino)butane]. 在 AIBN 引发剂的作用下, 得到 POL-dppm、POL-dppe
和 POL-dppb 聚合物; 进一步各聚合物与 $\mathrm{Rh}(\mathrm{CO})_{2}(\mathrm{acac})$ 络合, 得到相应的负载催化剂(Scheme 14).

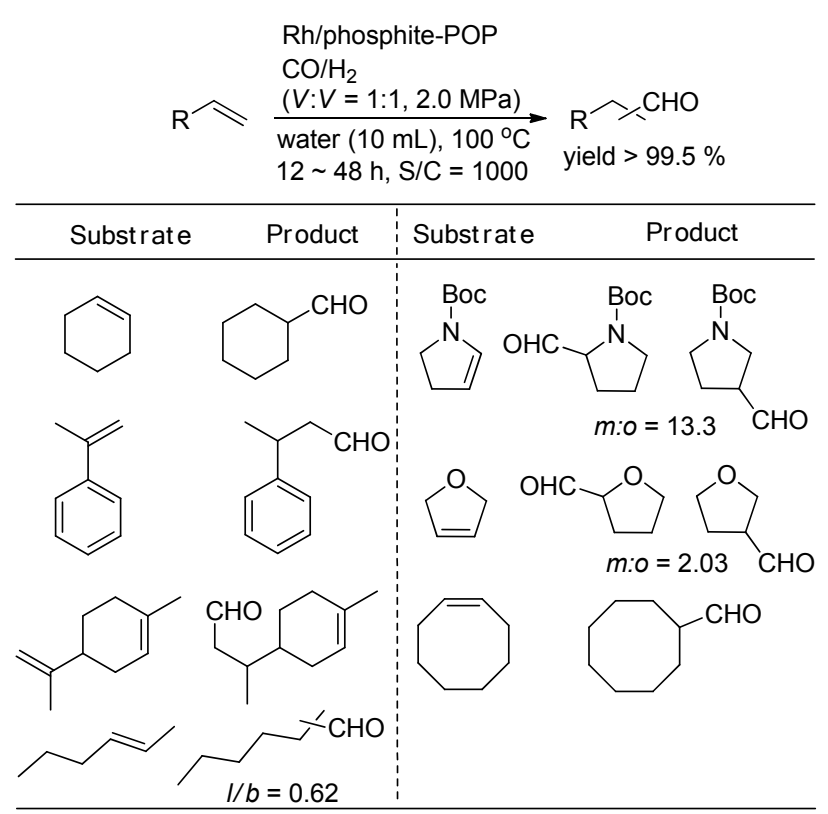

图式 $13 \mathrm{Rh} / \mathrm{phosphite-POP}$ 催化的烯烃氢甲酰化反应 Scheme $13 \mathrm{Rh} /$ phosphite-POP catalyzed hydroformylation of olefins

相比较 Rh/POL-dppm 和 Rh/POL-dppb, 催化剂 Rh/ POL-dppe 在烯烃的氢甲酰化反应中表现出更优秀的催 化性能. POL-dppe 主要由微孔和介孔组成, 且具有好的 热稳定性(表 1 , Entry 5); 该聚合物在反应溶剂中会发生 溶胀，可以使负载的 $\mathrm{Rh}$ 金属更好地分散在反应溶液中, 提高催化效率. 当以苯乙烯为反应底物时, 能以优秀的 转化率和高的区域选择性得到支链醛. 在催化剂的循环 测试中, Rh/POL-dppe 循环使用五次，均能保持稳定的 催化活性和选择性. 更重要的是, 该催化剂非常容易与 产物分离, 滤液经 ICP-OES 检测未发现铑的流失 $(<10$ $\mathrm{ppb}$ ). 另外, 在以长链的 1-辛烯和 1-十二烯的氢甲酰化 的反应中, 能以高的转化率和中等的区域选择性得到直 链产物 (Scheme 15). 在相同的反应条件下，与均相的 Rh/dppe 催化体系相比, Rh/POL-dppe 表现出更优秀的催 化性能.

\section{3 膦配体共聚物负载催化剂}

从以上的进展可以发现，虽然一系列高效的多孔聚 合物负载的铑催化剂用于烯烃的氢甲酰化反应，如

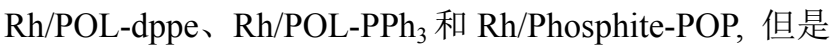
低的区域选择性仍然是需要解决的问题. 通过在乙烯基 三苯基膦的聚合物中嵌入大位阻的配体，如双齿膦配 体、亚磷酸酯配体和亚磷酰胺配体等，有助于明显提高 催化剂在氢甲酰化反应催化剂的活性和选择性. 在该类 膦配体共聚物中，三苯基膦单元常用来构建稳定的多孔 


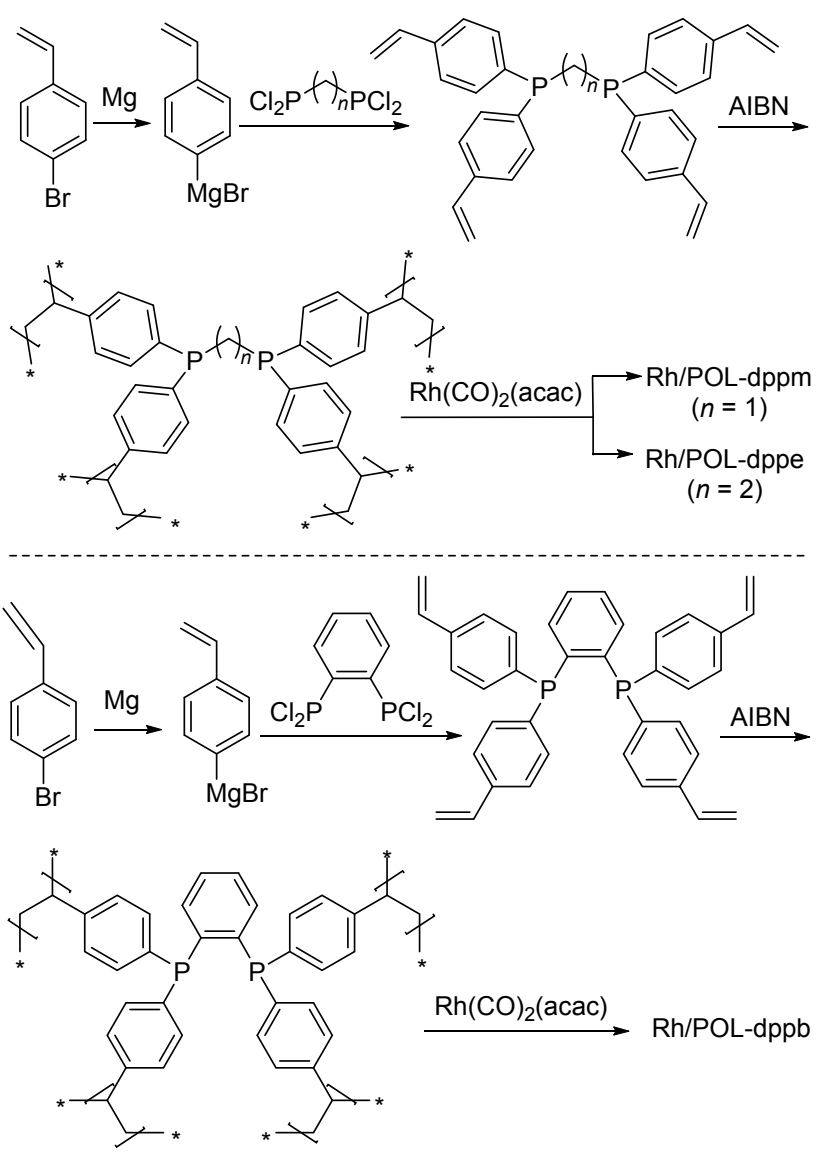

图式 $14 \mathrm{Rh} / \mathrm{POL}-\mathrm{dppm}$ 、Rh/POL-dppe 和 Rh/POL-dppb 的合 成

Scheme 14 Synthesis of Rh/POL-dppm, Rh/POL-dppe and $\mathrm{Rh} /$ POL-dppb

$$
\begin{aligned}
& \text { Rh/POL-dppe } \\
& \mathrm{R} \curvearrowright \frac{\mathrm{CO} / \mathrm{H}_{2}(\mathrm{~V}: \mathrm{V}=1: 1,2 \mathrm{MPa})}{\begin{array}{c}
\text { toluene }, 80 \text { or } 90^{\circ} \mathrm{C} \\
\mathrm{S} / \mathrm{C}=2000
\end{array}} \mathrm{R} \times \mathrm{CHO}^{\mathrm{CHO}} \\
& \begin{array}{ll}
\text { Styrene } & \text { conv. }=95.7 \%, b / l=7.93 \\
\text { 1-Octene } & \text { conv. }=96.9 \%, I / b=2.46 \\
\text { 1-Dodecene } & \text { conv. }=95.5 \%, I / b=2.45
\end{array}
\end{aligned}
$$

图式 $15 \mathrm{Rh} / \mathrm{POL}-d p p e$ 催化的烯烃氢甲酰化反应

Scheme $15 \mathrm{Rh} / \mathrm{POL}$-dppe catalyzed hydroformylation of olefins

结构; 嵌入的膦配体单元用来提高催化剂的活性和选择 性. 另外共聚物中的双膦配体和三苯基膦可以与铑同时 配位形成新的活性物种, 该协同作用有利于提高催化剂 的活性和选择性.

双膦配体 Xantphos 在均相的烯烃氢甲酰反应中有 着重要的应用. 2016 年, 丁云杰和严丽课题组 ${ }^{[34]}$ 合成了 乙烯基的 Xantphos 单体, 并将其与乙烯基 $\mathrm{PPh}_{3}$ 聚合得 到共聚物 POL-Xantphos\& $\mathrm{PPh}_{3}$ (Scheme 16). Rh/POLXantphos\& $\mathrm{PPh}_{3}$ 催化 $\mathrm{C}(6) \sim \mathrm{C}(12)$ 的末端烯烃的氢甲酰 化反应中, 能以高的催化活性(TOF: $300 \sim 500 \mathrm{~h}^{-1}$ )、化<smiles>CCCCc1cccc2c1Oc1c(CC)cccc1C2(C)c1cccc2c1Oc1c(CC)cccc1C2(C)C</smiles><smiles>C=Cc1ccc(P(c2ccc(C=C)cc2)c2cccc3c2Oc2c(P(c4ccc(C=C)cc4)c4ccc(C=C)cc4)cccc2C3(C)C)cc1</smiles>

图式 $16 \mathrm{Rh} / \mathrm{POL}-\mathrm{X} a n t p h o s \& \mathrm{PPh}_{3}$ 的合成

Scheme 16 Synthesis of Rh/POL-Xantphos\& $\mathrm{PPh}_{3}$

学选择性( $87 \%$ ～91\%)和区域区域选择性得到直链醛 (Scheme 17). 在 1-辛烯的反应中, 该催化剂可以循环使 用 5 次, 并能保持的催化活性和选择性. 更重要的是, 结合高角环形暗场扫描透射电子显微镜 (HAADFSTEM)和拓展 X 射线吸收精细结构谱图(EXAFS)分析表 明, 铑在固载催化剂中以单原子形态分散. 进一步综合 DFT (Discrete fourier transform)计算和实验数据对比, 表明在反应中铑同时与两种膦配体配位，形成了三膦配 位的活性中间体. 该催化物种有助于催化剂的稳定性和 选择性.

$\mathrm{R} / \mathrm{POL}-\mathrm{Xantphos} \& \mathrm{PPh}_{3}$
$\mathrm{CO} / \mathrm{H}_{2}(\mathrm{~V}: \mathrm{V}=1: 1,1.0 \mathrm{MPa})$
$\begin{array}{r}\text { toluene, } 100^{\circ} \mathrm{C}, 5 \mathrm{~h} \\ \mathrm{~S} / \mathrm{C}=5000\end{array}$

图式 $17 \mathrm{Rh} / \mathrm{POL}-\mathrm{X}$ antphos\& $\mathrm{PPh}_{3}$ 催化的烯烃氢甲酰化反应 Scheme $17 \mathrm{Rh} / \mathrm{POL}-\mathrm{Xantphos} \& \mathrm{PPh}_{3}$ catalyzed hydroformylation of olefins

相比较膦配体、亚磷酸酯磷配体和亚磷酰胺磷配体 具有强的 $\pi$ 酸性和大的空间效应，在烯烃的氢甲酰化反 应中, 可以得到更高的催化活性和区域选择性. 2016 年, 丁云杰和严丽课题组 ${ }^{[35]}$ 以 2-叔丁基-4-甲氧基苯酚为原 料, 经七步反应得到乙烯基 Biphenphos 单体; 该单体与 三乙烯基 $\mathrm{PPh}_{3}$ 共聚得到聚合物 CPOL-BP\& $\mathrm{PPh}_{3}$; 共聚 物进一步与 $\mathrm{Rh}(\mathrm{acac})(\mathrm{CO})_{2}$ 反应得到催化剂 $\mathrm{Rh} / \mathrm{CPOL}$ $\mathrm{BP} \& \mathrm{PPh}_{3}(\mathrm{Scheme} 18)$. 经表征, CPOL-BP\&PPh 3 具有高 的比表面积 $\left(643 \mathrm{~m}^{2} \cdot \mathrm{g}^{-1}\right)$ 、孔容 $\left(2.07 \mathrm{~cm}^{3} \cdot \mathrm{g}^{-1}\right)$ 和好的热稳 

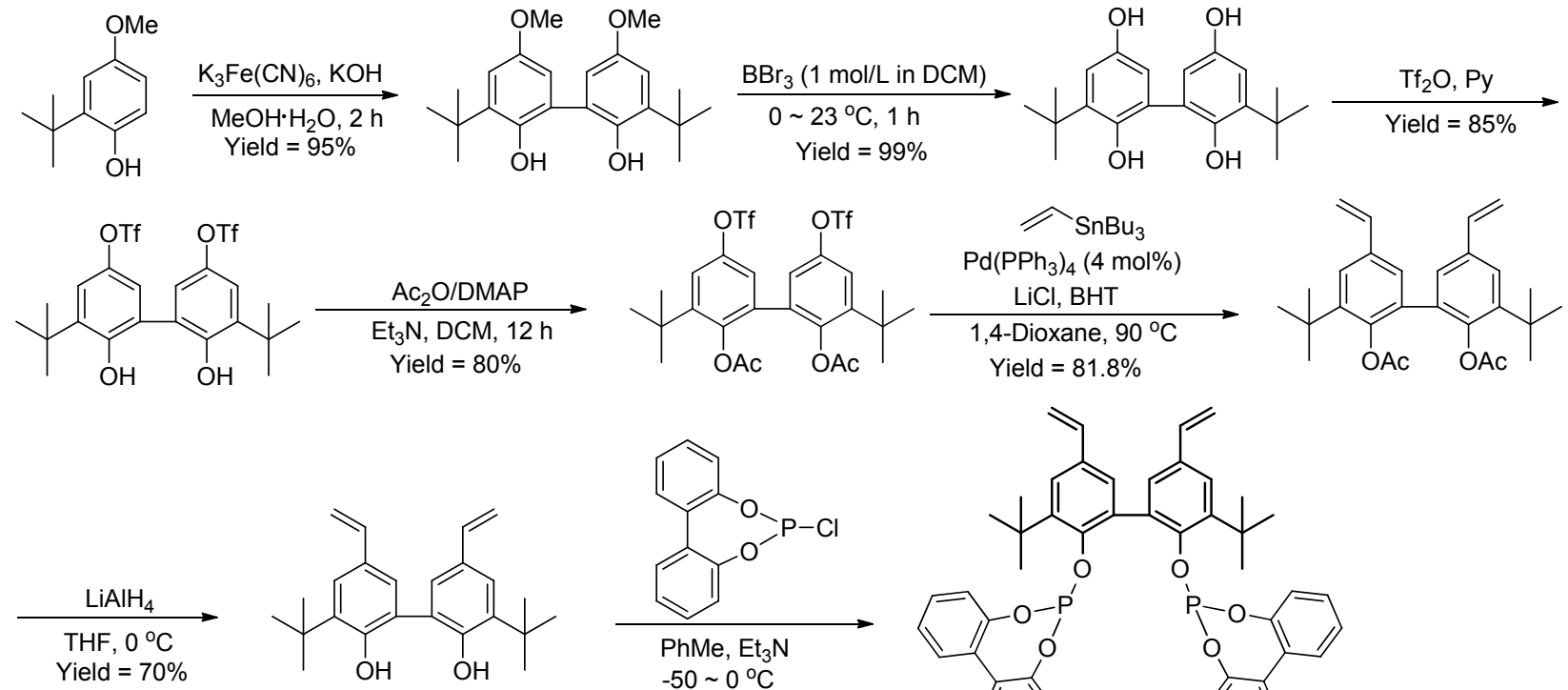

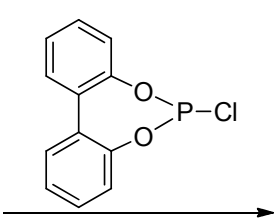

$\mathrm{PhMe}, \mathrm{Et}_{3} \mathrm{~N}$ $-50 \sim 0{ }^{\circ} \mathrm{C}$

Yield $=45 \%$<smiles>C=Cc1cc(C=C)c(O)c(-c2cc(C=C)cc(C(C)(C)C)c2O)c1</smiles>

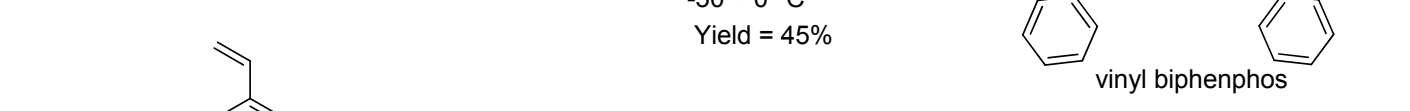

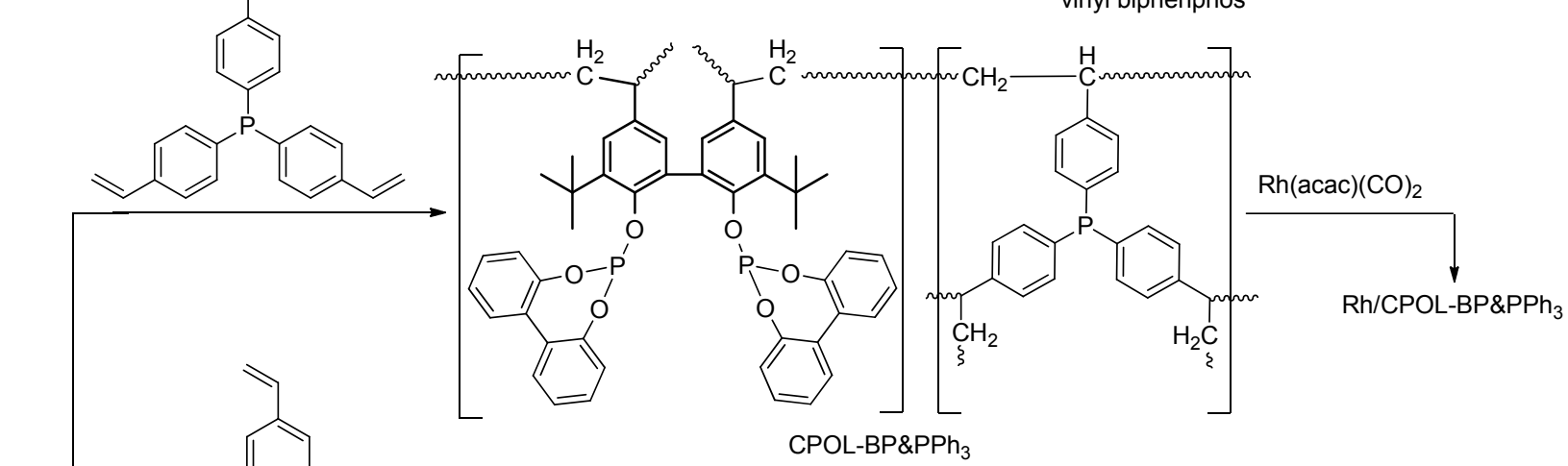

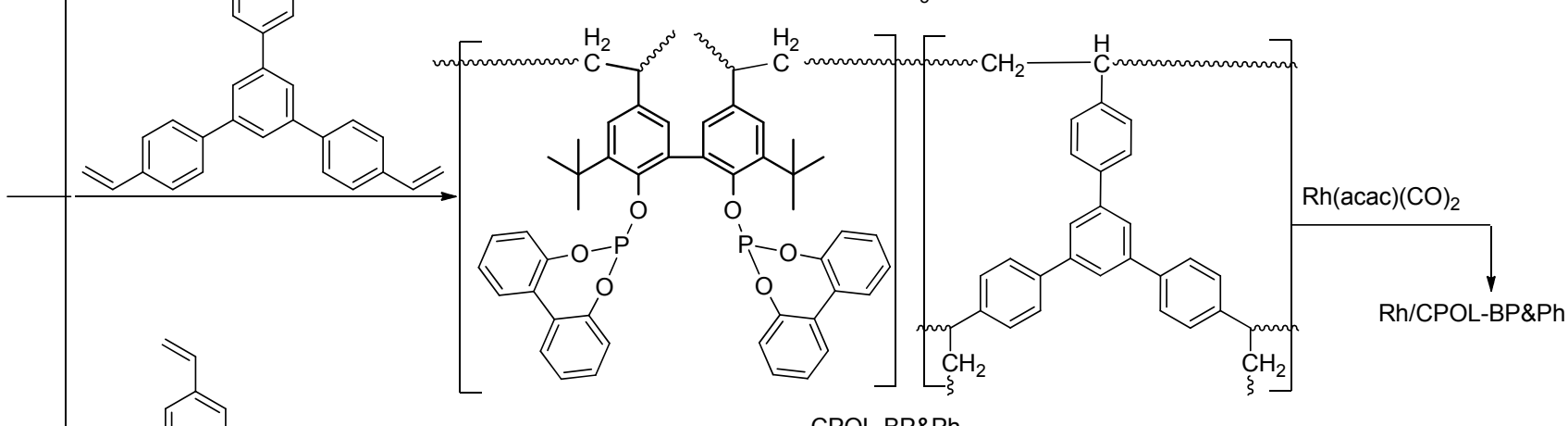
CPOL-BP\&Ph

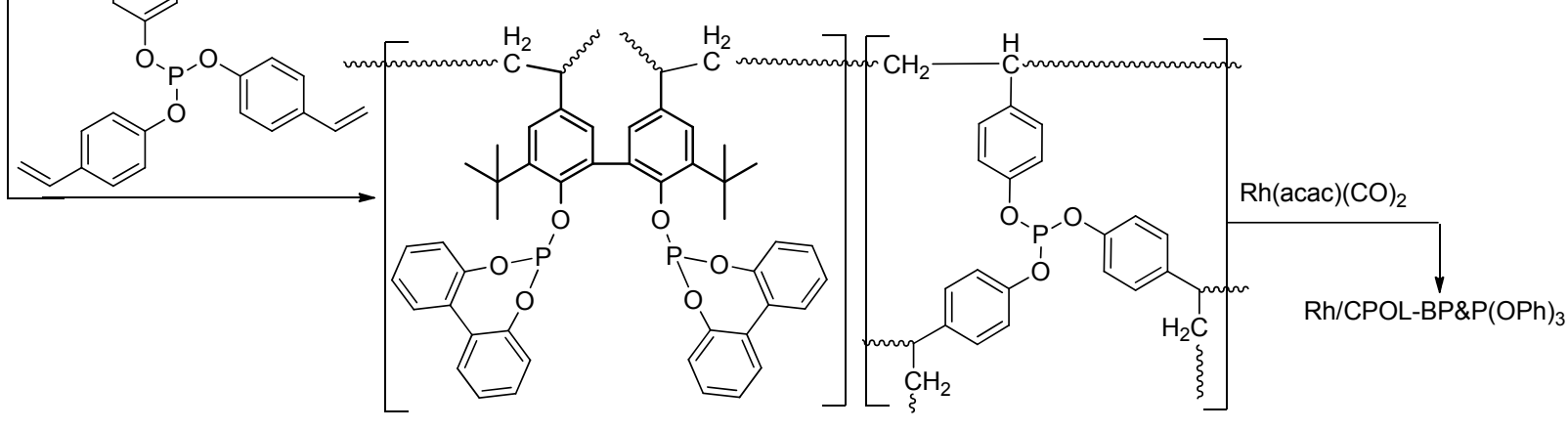

CPOL-BP\&P(OPh $)_{3}$

图式 $18 \mathrm{Rh} / \mathrm{CPOL}-\mathrm{BP} \& \mathrm{PPh}_{3} 、 \mathrm{Rh} / \mathrm{CPOL}-\mathrm{BP} \& \mathrm{Ph}$ 和 $\mathrm{Rh} / \mathrm{CPOL}-\mathrm{BP} \& \mathrm{P}(\mathrm{OPh})_{3}$ 的合成

Scheme 18 Synthesis of $\mathrm{Rh} / \mathrm{CPOL}-\mathrm{BP} \& \mathrm{PPh}_{3}, \mathrm{Rh} / \mathrm{CPOL}-\mathrm{BP} \& \mathrm{Ph}$ and $\mathrm{Rh} / \mathrm{CPOL}-\mathrm{BP} \& \mathrm{P}(\mathrm{OPh})_{3}$ 
定性(430 ${ }^{\circ} \mathrm{C}$, 表 1, Entry 11). Rh/CPOL-bp \& $\mathrm{PPh}_{3}$ 非均 相催化剂在末端烯烃氢甲酰化反应中表现出优秀的性 能, 以高的催化活性和优秀的区域选择性 $(l / b=96: 4 \sim$ $98 ： 2$ )得到相应的直链醛(Scheme 19); 催化性能甚至优 于 $\mathrm{Rh}$ /乙烯基 Biphenphos 催化体系. 另外, 该催化剂可 以循环使用六次, 且能保持稳定的催化活性和选择性. 值得注意的是, $\mathrm{Rh} / \mathrm{CPOL}-\mathrm{BP} \& \mathrm{PPh}_{3}$ 还能催化内烯烃的 氢甲酰化反应, 以高的区域选择性 $(l / b=92: 8 \sim 93: 7)$ 得到直链醛产物(Scheme 19). 之后, 丁云杰课题组将 $\mathrm{Rh} / \mathrm{CPOL}-\mathrm{BP} \& \mathrm{PPh}_{3}$ 应用于固定床反应器, 研究了其在 丁烯的氢甲酰化反应中的催化性能 ${ }^{[36]}$. 在 $\mathrm{Rh} / \mathrm{CPOL}-$ $\mathrm{BP} \& \mathrm{PPh}_{3}$ 摧毁的 1 -丁烯的反应中, 以优秀的催化活性 $\left(\mathrm{TOF}=11200 \mathrm{~h}^{-1}\right)$ 和优秀的区域选择性 $(l: b=62.2)$ 得到 直链的戊酫. 该催化剂在 100 到 $300 \mathrm{~h}$ 的连续反应中, 能 保持高的催化活性 $\left(\mathrm{TOF}=5400 \mathrm{~h}^{-1}\right)$ 和区域选择性 $(l$ : $b>61$ ). 为了研究催化剂单元结构对催化性能的影响, 还合成了 $\mathrm{Rh} / \mathrm{CPOL}-\mathrm{BP} \& \mathrm{Ph}$ 催化剂(Scheme 18). 在 $\mathrm{Rh} / \mathrm{CPOL}-\mathrm{BP} \& \mathrm{Ph}$ 催化的 1-已烯的氢甲酰化中, 反应时 间为 $100 \sim 300 \mathrm{~h}$ 内, TOF 值为 $2500 \mathrm{~h}^{-1}$ 且 $l: b$ 值从 45 降至 18. 通过实验对比及催化剂结构表征, Rh/CPOL-

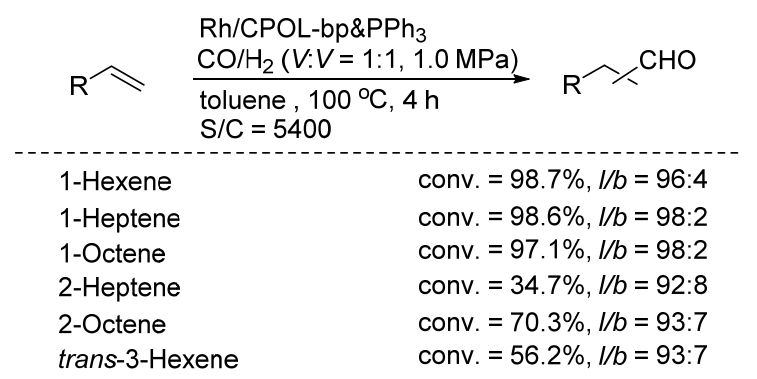

图式 $19 \mathrm{Rh} / \mathrm{CPOL}-\mathrm{BP} \& \mathrm{PPh}_{3}$ 催化的烯烃氢甲酰化反应 Scheme $19 \mathrm{Rh} / \mathrm{CPOL}-\mathrm{BP} \& \mathrm{PPh}_{3}$ catalyzed hydroformylation of olefins
$\mathrm{BP} \& \mathrm{PPh}_{3}$ 优秀的催化性能与两种不同膦配体(Bi-phenphos 和 $\mathrm{PPh}_{3}$ )和铑金属的协同作用有关.

2018 年, 丁云杰和严丽课题组 ${ }^{[37]}$ 将乙烯基 Biphenphos 和乙烯基的三苯基亚磷酸酯配体聚合得到 CPOL$\mathrm{BP} \& \mathrm{P}(\mathrm{OPh})_{3}(\mathrm{Scheme} 18)$, 并研究了该类聚合物负载铑 催化剂在氢甲酰化反应中的应用. 在 1-丁烯为底物反应 中, 该催化剂能以高的活性 $\left(\mathrm{TOF}=2490.3 \mathrm{~h}^{-1}\right)$ 和区域选 择性 $(l: b=40.0)$ 得到直链戊醛. 作者进一步通过在线 FT-IR 考察了反应中催化剂物种的变化. 经实验发现, 在聚合物负载催化反应过程中，铑金属更倾向同时与不 同类型的膦(Biphenphos 和 $\left.\mathrm{P}(\mathrm{OPh})_{3}\right)$ 配位形成新的 $\mathrm{Rh}-\mathrm{H}$ 物种 $(\mathrm{Scheme} 20)$. 当 $\mathrm{Rh} / \mathrm{CPOL}-\mathrm{BP} \& \mathrm{P}(\mathrm{OPh})_{3}$ 应用于固定 床反应器测试时, 反应 $36 \mathrm{~h}$ 后, TOF 值从 4626 降至 2806 $\mathrm{h}^{-1}$; 但反应 $24 \mathrm{~h}$ 后, $l: b$ 值从 18.6 升高到 49.4. 进一步 证明反应中逐渐形成的 Rh-H 催化物种对区域选择性的 提高有重要的决定作用.<smiles>O=C[PH]1CCPPP1</smiles><smiles>O=C[PH3+]([O-])(P)P</smiles><smiles>[O-][Pb]([O-])([O-])[PH-]</smiles>

$\mathrm{P}$ : Biphenphos, $\mathrm{P}^{\prime}: \mathrm{P}(\mathrm{OPh})_{3}$; Ligands in the polymer skeleton

图式 $20 \mathrm{Rh} / \mathrm{CPOL}-\mathrm{BP} \& \mathrm{P}(\mathrm{OPh})_{3}$ 催化的氢甲酰化反应中形成 的 $\mathrm{Rh}-\mathrm{H}$ 物种

Scheme $20 \mathrm{Rh}-\mathrm{H}$ species in the $\mathrm{Rh} / \mathrm{CPOL}-\mathrm{BP} \& \mathrm{PPh}_{3}$ catalyzed hydroformylation of olefins

我们课题组 ${ }^{[38]}$ 发展了一类双齿亚磷酰胺配体与 $\mathrm{PPh}_{3}$ 聚合物，将其负载铑金属后得到 $\mathrm{Rh} / \mathrm{POL}-\mathrm{BPa} \&$ $\mathrm{PPh}_{3}$. 以 2,2'-二羟基联苯为原料，经溴化反应、羟基保 护、Suzuki 偶联等五步反应得到乙烯基双齿亚磷酰胺配 体单体(Scheme 21). Rh/POL-BPa\&PPh 3 具有高的表面积

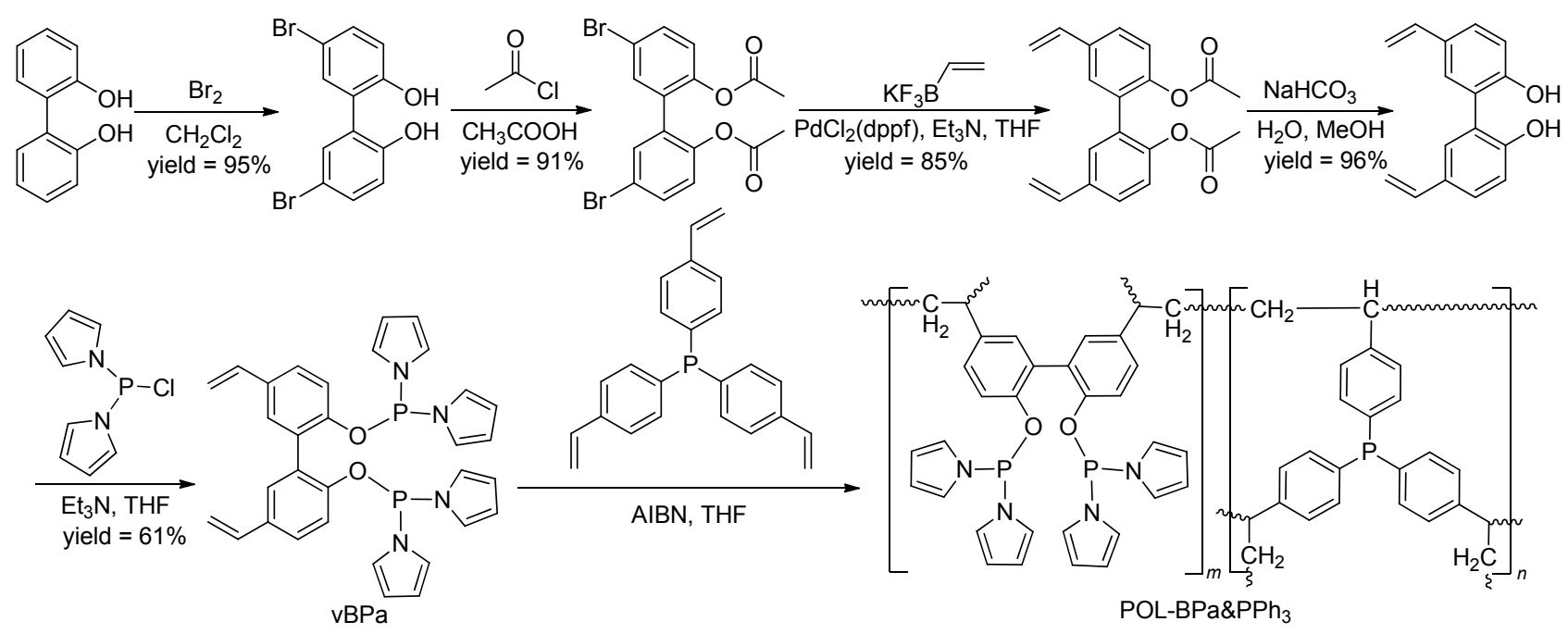

图式 $21 \mathrm{POL}-\mathrm{BPa} \& \mathrm{PPh}_{3}$ 的合成

Scheme 21 Synthesis of POL-BPa\& $\mathrm{PPh}_{3}$ 
和热稳定性(表 1, Entry 16). 该催化剂在末端烯烃的氢 甲酰化反应中表现出优秀的活性、区域选择性 $(l / b=$ 12.1 52.8)和循环性(Scheme 22a). 在 1-己烯为底物的 反应中, $\mathrm{TON}$ 最高可达 $45.3 \times 10^{4}$; 且催化剂可以循环使 用 10 次, 并能保持反应活性和区域选择性. 通过实验对 比, $\mathrm{Rh} / \mathrm{POL}-\mathrm{BPa} \& \mathrm{PPh}_{3}$ 的催化性能要优于 $\mathrm{Rh} / \mathrm{POL}-\mathrm{PPh}_{3}$ 催化剂, 说明聚合物中双齿亚磷酰胺配体单元对催化剂 的性能提高有着重要的影响. 另外, 该类催化剂在内烯 烃的异构化/氢甲酰化反应中也表现出优秀的催化性能 (Scheme 22b).

(a)

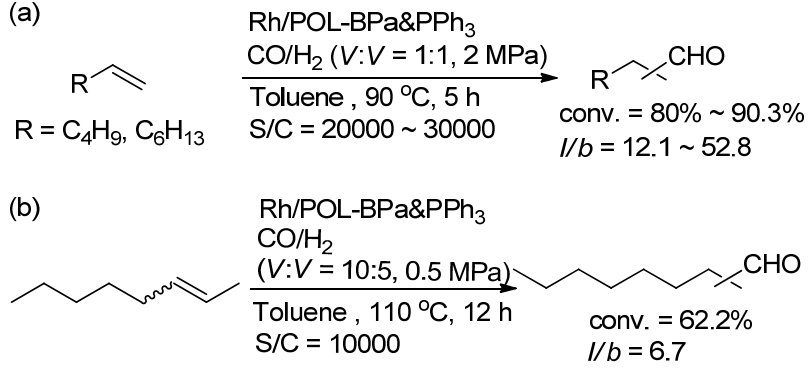

图式 $22 \mathrm{Rh} / \mathrm{CPOL}-\mathrm{BPa} \& \mathrm{PPh}_{3}$ 催化的烯烃氢甲酰化反应 Scheme $22 \mathrm{Rh} / \mathrm{CPOL}-\mathrm{BPa} \& \mathrm{PPh}_{3}$ catalyzed hydroformylation of olefins

基于以上的研究基础, 我们进一步将该类催化剂用 于非均相的炔烃氢甲酰化反应研究. 与烯烃相比, 炔烃 氢甲酰化反应表现出低的催化活性、难以控制的化学选 择性和区域选择性 ${ }^{[39]}$. 首先合成了以联萗为骨架的乙 烯基亚磷酸酰胺单体，并与乙烯基三苯基膦聚合得到

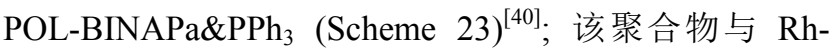
$(\mathrm{acac})(\mathrm{CO})_{2}$ 配位络合得到 $\mathrm{Rh} / \mathrm{POL}-\mathrm{BINAPa} \& \mathrm{PPh}_{3}$ 催化 剂. $\mathrm{Rh} / \mathrm{POL}-\mathrm{BINAPa} \& \mathrm{PPh}_{3}$ 在炔烃的氢甲酰化反应中, 该类催化剂表现出优秀的催化活性、立体选择性和化学 选择性(Scheme 24). 在二苯乙炔为底物的氢甲酰化反 应中, 催化剂用量最低可降至五万分之一. 产物不饱和 醛的 $E / Z$ 构型比值高达 $40 ： 1$. 该催化体系对各种官能 团具有兼容性，如甲基、甲氧基、醛基、溴基. 另外，杂 芳环底物也能顺利转化得到相应的不饱和醛. 该类催化 剂也能高效地催化烷基炔, 以高的收率得到烷基不饱和 醛. $\mathrm{Rh} / \mathrm{POL}-\mathrm{BINAPa} \& \mathrm{PPh}_{3}$ 在苯乙炔的循环催化测试中 可重复使用十次, 且没有检测到铑的损失. 值得注意的 是, $\mathrm{Rh} / \mathrm{POL}-\mathrm{BINAPa} \& \mathrm{PPh}_{3}$ 的催化活性要高于均相 $\mathrm{Rh} / \mathrm{BINAPa} / \mathrm{PPh}_{3}$ 体系. 我们推测在反应中双齿亚磷酸 酰胺配体和三苯基膦与铑配位, 得到三膦配位的 $\mathrm{Rh}-\mathrm{H}$ 活性中间体 B. 与中间体 $\mathbf{A}$ 相比, 中间体 $\mathbf{B}$ 铑周围的空 间位阻较大, 使得其在炔烃的氢甲酰化反应中表现出低 的催化活性(Scheme 25). 在聚合物中, 由于配体单体固 定在聚合链中，降低了两种配体之间与铑的协同效应，
因此负载后的催化剂具有更高的催化活性.

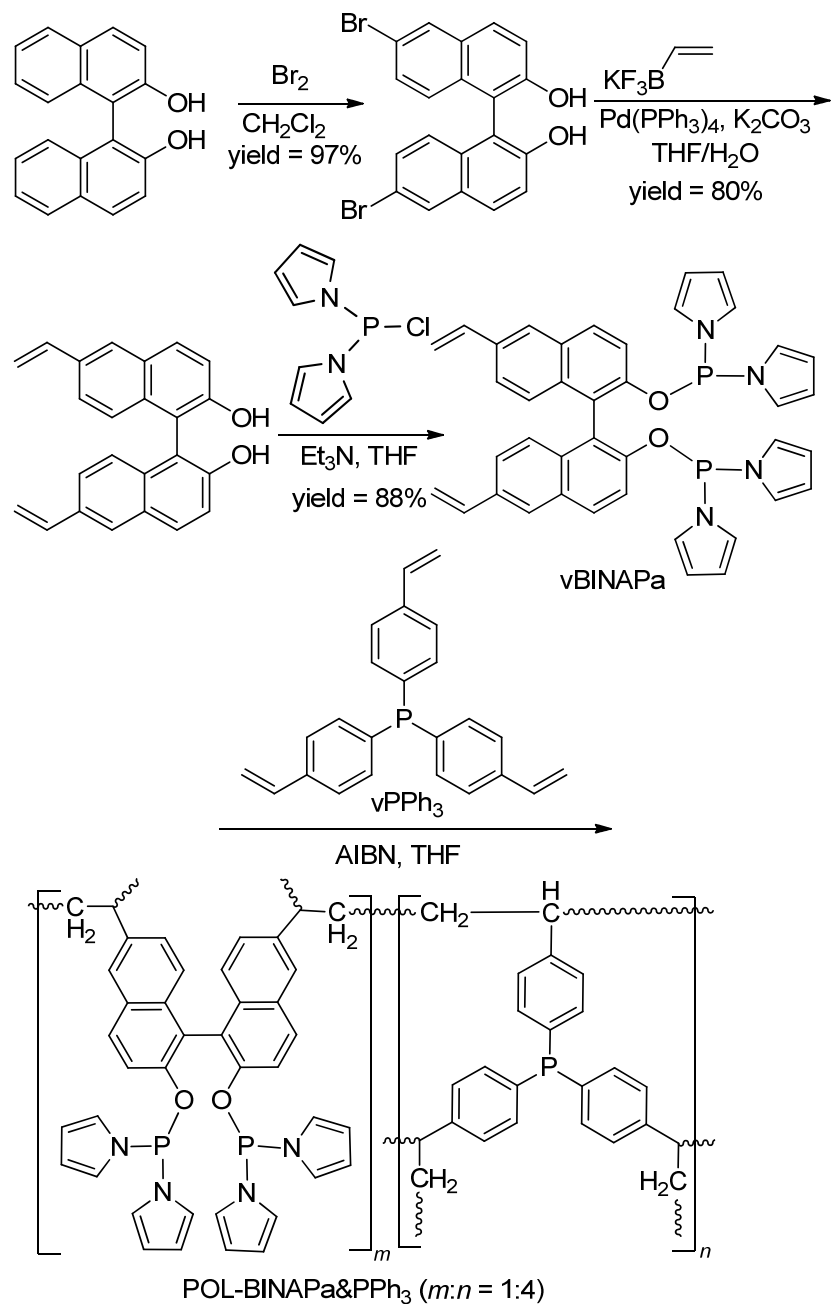

图式 23 POL-BINAPa\& $\mathrm{PPh}_{3}$ 的合成 Scheme 23 Synthesis of POL-BINAPa\& $\mathrm{PPh}_{3}$

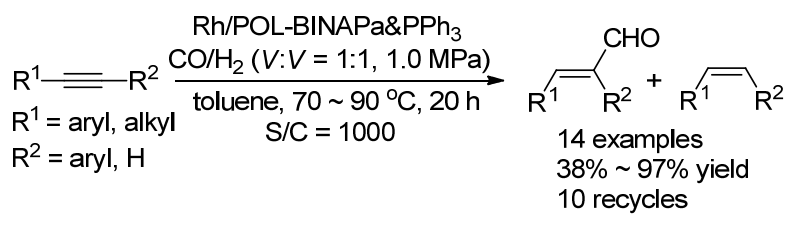

图式 24 POL-BINAPa\& $\mathrm{PPh}_{3}$ 的应用

Scheme 24 Application of POL-BINAPa\& $\mathrm{PPh}_{3}$

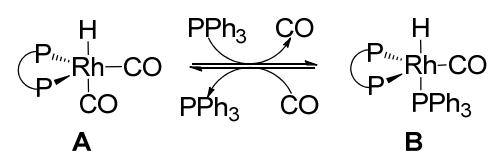

图式 25 催化物种 $\mathbf{A}$ 和 $\mathbf{B}$

Scheme 25 Catalytically active species A and B

\section{4 离子型聚合物负载催化剂}

在氢甲酰化反应中，以水作为反应溶剂，采用水溶 性的离子型膦配体，可以实现催化剂和产物分离，以及 
催化剂的循环使用. Ruhrchemie/Rhône-Poulenc 作为代 表型工艺, 水溶性 TPPTS 配体用于丙烯的氢甲酰化反 应. 但对于水溶性差的长链烯烃, 该类工艺表现出低的 反应性能. 肖丰收课题组和马胜前课题组 ${ }^{[41]}$ 发展的离 子型多空聚合物, 可以有效地解决该类问题. 首先将阳 离子的 4-乙烯基芐基乙烯基三苯基膦与阴离子的磺酸 基膦配体结合; 进一步在 AIBN 的催化下聚合乙烯基官 能团得到离子型多孔有机膦配体聚合物. 通过嵌入不同 的阴离子配体可以分别得到 PSA-PPh 3 和 PSA-Xantphos, 再负载铑金属后得到相应的催化剂(Scheme 26) ${ }^{[41]}$. 该 类催化剂具有两亲性, 不仅具有亲水性, 而且与烯烃也 具有很好的相容性. 以水为溶剂, $\mathrm{Rh} / \mathrm{PSA}-\mathrm{PPh}_{3}$ 和 $\mathrm{Rh} / \mathrm{PSA}$ - Xantphos 催化 1-辛烯的氢甲酰化反应, 分别以 $96.3 \%$ 和 $94.7 \%$ 的转化率及 2.8 和 39 的 $l / b$ 值得到相应的 醛产物(Scheme 27). 而以 Rh/TPPTS 作为催化剂的反应, 仅得到 29.7\%转化率和 2.9 正异比. 该工作为水相催化 烯烃的氢甲酰化反应的催化剂设计开辟了新的方向.

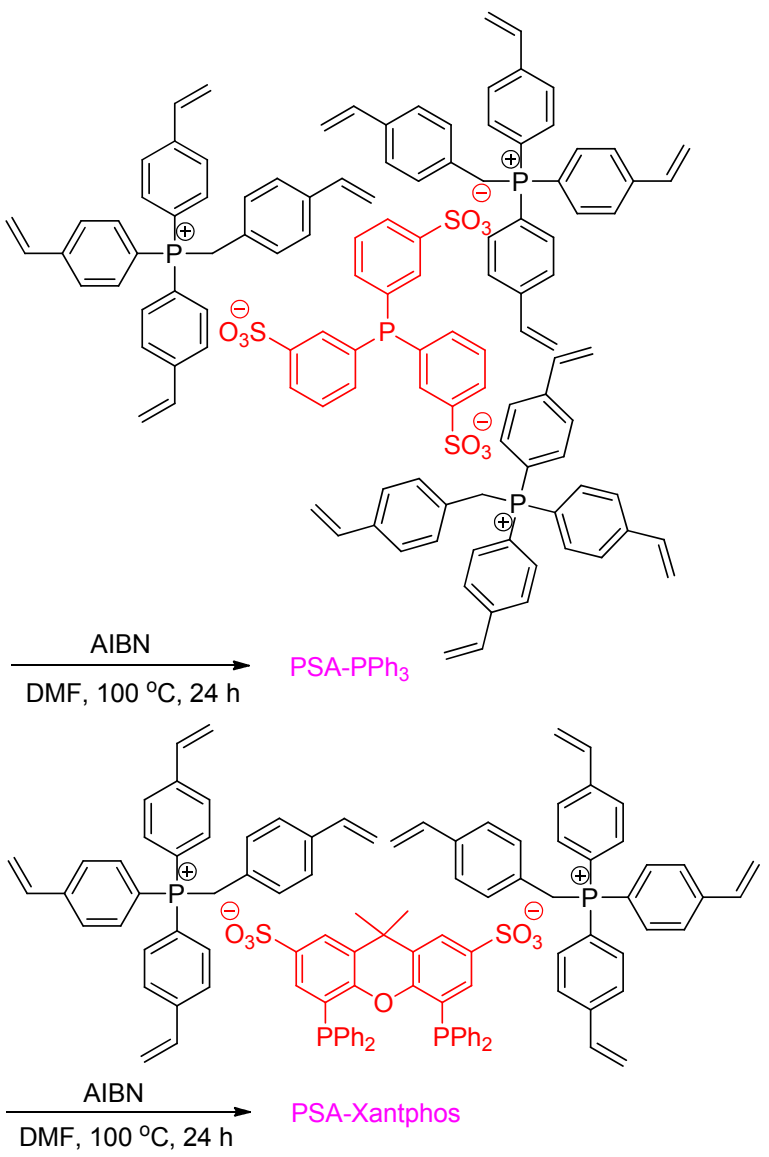

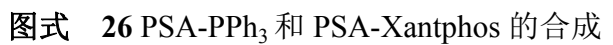
Scheme 26 Synthesis of PSA-PPh ${ }_{3}$ 和 PSA-Xantphos

\section{3 结论与展望}

从以上的结果可以发现, 经过近二十年的研究, 聚

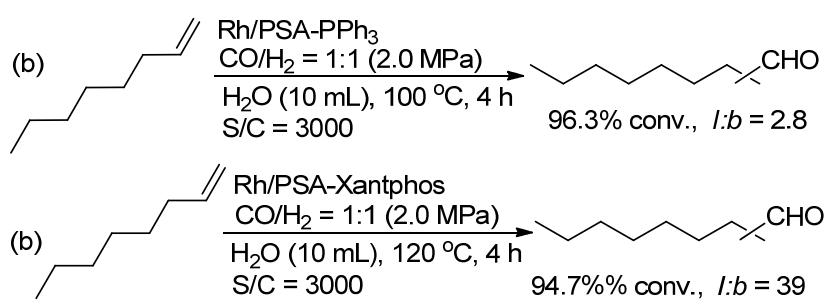

图式 $27 \mathrm{Rh} / \mathrm{PSA}-\mathrm{PPh}_{3}$ 和 $\mathrm{Rh} / \mathrm{PSA}-\mathrm{X} a n t p h o s$ 催化的 1-辛烯氢 甲酰化反应

Scheme $27 \mathrm{Rh} / \mathrm{PSA}-\mathrm{PPh}_{3}$ and $\mathrm{Rh} / \mathrm{PSA}-\mathrm{Xantphos}$ catalyzed hydroformylation of 1-octene

合物负载催化剂的发展及其应用已经取得很大的进展. 特别是近几年，由有机配体单体构建的多孔聚合物出 现，为固载催化剂的发展提供了新的契机. 该方法不仅 可以把单齿和双齿膦配体嵌入到聚合物链中，而且可以 把不同类型的配体修饰到多孔聚合物中，包括膦配体、 亚磷酸酯配体、亚磷酰胺配体和离子型膦配体. 发展的 多孔聚合物负载催化剂具有高的比表面积和好的稳定 性; 在氢甲酰化反应的应用中，该类多相催化剂可以取 得与均相体系一样甚至更加优异催化效果. 更重要的 是，由配体构建的聚合物结构可以有效避免铑的流失， 使催化剂具有好的循环性. 基于实验结果和材料的优秀 性能，都为多孔聚合物负载催化剂的工业化奠定了基 础. 与无机物固载催化剂相比, 多孔有机聚合物负载催 化剂在活性、选择性和稳定性方面都表现出更优的性能. 因此, 多孔有机聚合物针定铑的多相催化剂可能成为非 均相氢甲酰化反应催化剂的重要研究方向.

尽管多孔聚合物负载催化剂的研究工作取得了一 定得进展，但仍存在许多亟待解决的问题：(1)进一步探 索聚合物中配体和铑的配位关系及催化剂活性中间体, 为提高催化剂性能和设计新型催化剂提供理论基础; (2) 多孔聚合物的孔径主要分布在微孔和介孔范畴. 孔径的 大小与催化活性、选择性及反应底物的影响关系需要进 一步探索; (3)非均相氢甲酰化催化剂的研究主要集中于 非手性催化剂, 手性聚合物负载催化剂发展和应用仍是 一个具有挑战和潜力的研究方向 ${ }^{[42]} ;(4)$ 目前探索的底物 相对比较狭窄, 因此有必要进一步拓展烯烃底物的范 围.

另外, 目前聚合物负载催化剂在实验室取得了一定 的成功，但该类催化剂在工业化过程中仍然有很多问题 需要解决: (1)氢甲酰化反应是一个放热反应，但聚合物 载体的热传导性较差, 反应体系内部温度过高将会影响 催化剂的活性和选择性，甚至导致催化剂的钝化或失 活; (2)相比较无机载体, 聚合物载体的机械强度较差, 这样会增加该类催化剂在使用中的损耗; (3)聚合物负载 催化剂的合成步骤较多，势必会导致 “三废” 问题的产 
生. 另外, 合成过程中对无水无氧条件要求严格. 这些 都会影响到催化剂的大量生产.

因此，在铑催化的非均相的氢甲酰化反应研究中， 新型高效多孔聚合物负载催化剂的研究不仅是一个具 有挑战性和发展潜力的方向, 而且具有巨大的应用前 景.

\section{References}

[1] (a) van Leeuwen, P. W. N. M.; Claver, C. Rhodium catalyzed hydroformylation, Kluwer Academic Publishers, Dordrecht, 2000.

(b) Haumann, M.; Riisager, A. Chem. Rev. 2008, 108, 1474.

(c) Hebrard, F.; Kalck, P. Chem. Rev. 2009, 109, 4272.

(d) Franke, R.; Selent, D.; Borner, A. Chem. Rev. 2012, 112, 5675.

(e) Pospech, J.; Fleischer, I.; Franke, R.; Buchholz, S.; Beller, M. Angew. Chem., Int. Ed. 2013, 52, 2852.

[2] Roelen, O. U. S. 2327066,1943 [Chem. Abstr. 1944, 38, 363].

[3] Börner, A.; Franke, R. Hydroformylation: Funda Mentals, Processes, and Applications in Organic Synthesis, Wiley-VCH, Weinheim, 2016.

[4] (a) Casey, C. P.; Paulsen, E. L.; Beuttenmueller, E. W.; Proft, B. R.; Petrovich, L. M.; Matter, B. A.; Powell, D. R. J. Am. Chem. Soc. 1997, 119, 11817.

(b) Herrmann, W. A.; Kohlpaintner, C. W.; Herdtweck, E.; Kiprof, P. Inorg. Chem. 1991, 30, 4271.

(c) Yu, S.; Zhang, X.; Yan, Y.; Cai, C.; Dai, L.; Zhang, X. Chem. Eur. J. 2010, 16, 4938.

(d) Yan, Y.; Zhang, X.; Zhang, X. Adv. Synth. Catal. 2007, 349, 1582.

(e) Chen, C.; Li, P.; Hu, Z.; Wang, H.; Zhu, H.; Hu, X.; Wang, Y.; Lv, H.; Zhang, X. Org. Chem. Front. 2014, 1, 947.

[5] Klein, H.; Jackstell, R.; Wiese, K.-D.; Borgmann, C.; Beller, M. Angew. Chem., Int. Ed. 2001, 40, 3408.

[6] (a) Carbó, J. J.; Maseras, F.; Bo. C.; van Leeuwen, P. W. N. M. J. Am. Chem. Soc. 2001, 123, 7630.

(b) Kranenburg, M.; van der Burgt, Y. E. M.; Kamer, P. C. J.; van Leeuwen, P. W. N. M.; Goubitz, K.; Fraanje, J. Organometallics 1995, 14, 3081.

(c) Van der Veen, L. A.; Boele, M. D. K.; Bregman, F. R.; Kamer, P. C. J.; van Leeuwen, P. W. N. M.; Goubitz, K.; Fraanje, J.; Schenk, H.; Bo, C. J. Am. Chem. Soc. 1998, 120, 11616.

(d) van der Veen, L. A.; Kamer, P. C. J.; van Leeu wen, P. W. N. M. Angew. Chem., Int. Ed. 1999, 38, 336.

[7] Burke, P. M.; Garner, J. M.; Kreutzer, K. A.; Teunis sen, A. J. J. M.; Snijder, C. S.; Hansen, C. B. WO 97/33854, 1997.

[8] (a) Cunny, G. D.; Buchwald, S. L. J. Am. Chem. Soc. 1993, 115, 2066.

(b) Behr, A.; Obst, D.; Schulte, C. J. Mol. Catal. A-Chem. 2003, 206, 179.

[9] (a) van der Slot, S. C.; Duran, J.; Luten, J.; Kamer, P. C. J.; van Leeuwen, P. W. N. M. Organometallics 2002, 213873.

(b) Yan, Y.; Zhang, X.; Zhang, X. J. Am. Chem. Soc. 2006, 128, 16058.

(c) Yu, S.; Chie, Y.; Guan, Z.; Zou, Y.; Li, W.; Zhang, X. Org. Lett. 2009, 11,241

(d) Jia, X.; Wang, Z.; Xia, C.; Ding, K. Chem. Eur. J. 2012, 18, 15288.

(e) Ren, X.; Zheng, Z.; Zhang, L.; Wang, Z.; Xia, C.; Ding, K. Angew. Chem., Int. Ed. 2017, 56, 310.

(f) Jia, X.; Ren, X.; Wang, Z.; Xia, C.; Ding, K. Chin. J. Org. Chem. 2019, 39, 207 (in Chinese)

(贾肖飞, 任新意, 王正, 夏春谷, 丁奎岭, 有机化学, 2019, 39, 207.)

(g) Chen, C.; Qiao, Y.; Geng, H.; Zhang, X. Org. Lett. 2013, 15, 1048.
[10] (a) Li, C; Wang, W; Yan, L.; Ding, Y. Front. Chem. Sci. Eng. 2018, $12,113$.

(b) Zhang, J.; Sun, P.; Zhao, Z. L.; Li, F. W. Chin. Sci. Bull. 2019, 64, 3173 .

[11] (a) Arhancet, J. P.; Davis, M. E.; Merola, J. S.; Hanson, B. E. Nature 1989, 339, 454.

(b) Chaudhari, R. V.; Bhanage, B. M.; Deshpande, R. M. Nature 1995, 373, 501 .

(c) Sharma, S. K.; Jasra, R. V. Catal. Today 2015, 247, 70.

(d) Hapiot, F.; Ponchel, A.; Tilloy, S.; Monflier, Compt. Rend. Chim. 2011, 14, 149.

(e) Paganelli, S.; Piccolo, O.; Pontini, P.; Tassini, R.; Rathod, V. D. Catal. Today 2015, 247, 64.

[12] (a) Horváth, I. T.; Kiss, G.; Cook, R. A., Bond, J. E.; Stevens, P. A.; Rábai, J.; Mozeleski, E. J. J. Am. Chem. Soc. 1998, 120, 3133.

(b) Cornils, B. Angew. Chem., Int. Ed. 1997, 36, 2057.

(c) Chen, W. P.; Xu, L. J.; Xiao, J. L. Chem. Commun. 2000, 10, 839.

(d) Horvath, I. T.; Rabai, J. Science 1994, 266, 72.

[13] (a) Mehnert, C. P.; Cook, R. A.; Dispenziere, N. C.; Afeworki, M. J. Am. Chem. Soc. 2002, 124, 12932.

(b) Riisager, A.; Fehrmann, R.; Flicker, S.; van Hal, R.; Haumann, M.; Wasserscheid, P. Angew. Chem., Int. Ed. 2005, 44, 815.

(c) Jin, X.; Feng, J.; Ma, Q.; Song, H.; Liu, Q.; Xu, B.; Zhang, M.; Li, S.; Yu, S. Green Chem. 2019, 21, 3267.

(d) Walter, S.; Spohr, H.; Franke, R.; Hieringer, W.; Wasserscheid, P.; Haumann, M. ACS Catal. 2017, 7, 1035.

[14] (a) David, J.; Cole-Hamilton, O. J. Science 2003, 299, 1702.

(b) Jessop, P. G.; Hsion, Y.; Ikariya, T.; Noyori, R. J. Am. Chem. Soc. 1996, 118, 344

(c) Kainz, S., Koch, D.; Baumann, W.; Leitner, W. Angew. Chem., Int. Ed. 1997, 36, 1628.

(d) Koeken, A. C. J.; Smeets, N. M. B. Catal. Sci. Technol. 2013, 3, 1036.

(e) Estorach, C. T.; Orejon, A.; Masdeu-Bulto, A. M. Green Chem. 2008, 10, 545 .

[15] Gärtner, L.; Cornils, B.; Lappe, P. (to Ruhrchemie AG) EP 0107006, 1983 [Chem. Abstr. 1984, 101, 55331].

[16] (a) Kuntz, E. G. CHEMTECH 1987, 17, 570.

(b) Cornils, B.; Kuntz, E. G. J. Organomet. Chem.1995, 502, 177.

[17] Herrmann, W. A.; Kohlpaintner, C. W.; Bahrmann, H.; Konkol, W. J. Mol. Catal. 1992, 73, 191.

[18] Bahmann, H.; Bergrath, K.; Kleiner, H.-J.; Lappe, P.; Naumann, C.; Peters, D.; Regnat, D. J. Organomet. Chem. 1996, 520, 97.

[19] (a) Vunain, E.; Ncube, P.; Jalama, K.; Meijboom, R. J. Porous Mater. 2018, 25, 303

(b) Malihan, L. B.; Nisola, G. M.; Mittal, N.; Lee, S.-P.; Seo, J. G.; Kim, H.; Chung, W. J. RSC Adv. 2016, 6, 33901.

(c) Sudheesh, N.; Parmar, J. N.; Shukla, R. S. Appl. Catal. A Gen. 2012, 415, 124.

(d) Yan, L.; Ding, Y. J.; Lin, L. W.; Zhu, H. J.; Yin, H. M.; Li, X. M.; Lu, Y. J. Mol. Catal. A-Chem. 2009, 300, 116.

[20] (a) Wolf, P.; Logemann, M.; Schorner, M.; Keller, L.; Haumann, M.; Wessling, M. RSC Adv. 2019, 9, 27732.

(b) Weiss, A.; Munoz, M.; Haas, A.; Rietzler, F.; Steinruck, H.-P.; Haumann, M.; Wasserscheid, P.; Etzold, B. J. ACS Catal. 2016, 6, 2280 .

(c) Weiß, A.; Giese, M.; Lijewski, M.; Franke, R.; Wasserscheid, P.; Haumann, M. Catal. Sci. Technol. 2017, 7, 5562.

[21] (a) Chuai, H. Y.; Su, P.; Liu, H.; Zhu, B.; Zhang, S.; Huang, W. Catalysts 2019, 9, 194.

(b) Liu, J.; Yan, L.; Ding, Y.; Jiang, M.; Dong, W.; Song, X.; Liu, T.; Zhu, H. Appl. Catal. A Gen. 2015, 492, 127.

[22] (a) Nozaki, K.; Itoi, Y.; Shibahara, F.; Shirakawa, E.; Ohta, T.; Takaya, H.; Hiyama, T. J. Am. Chem. Soc. 1998, 120, 4051.

(b) Nozaki, K.; Shibahara, F.; Itoi, Y.; Shirakawa, E.; Ohta, T.; Takaya, H.; Hiyama, T. Bull. Chem. Soc. Jpn. 1999, 72, 1911.

[23] (a) Shibahara, F.; Nozaki, K.; Hiyama, T. J. Am. Chem. Soc. 2003, 
125,8555 .

(b) Nozaki, K.; Shibahara, F.; Hiyama, T. Chem. Lett. 2000, 694.

[24] (a) Stiriba, S. E.; Slagt, M. Q.; Kautz, H.; Klein Gebbink, R. J. M.; Thomann, R.; Frey, H.; van Koten, G. Chem. Eur. J. 2004, 10, 1267. (b) Kumar, K. R.; Kizhakkedathu, J. N.; Brooks, D. E. Macromol. Chem. Phys. 2004, 205, 567

(c) Wilms, D.; Stiriba, S. E.; Frey, H. Acc. Chem. Res. 2010, 43, 129

(d) Slagt, M. Q.; Stiriba, S.-E.; Kautz, H.; Klein Gebbink, R. J. M.; Frey, H.; van Koten, G. Organometallics 2004, 23, 1525.

[25] Ricken, S.; Osinski, P. W.; Eilbracht, P.; Haag, R. J. Mol. Catal. A-Chem. 2006, 257, 78 .

[26] (a) Wang, H.; Sun, W.; Xia, C. J. Mol. Catal. A: Chem. 2003, 206, 199.

(b) Makhubela, B. C. E.; Jardine, A.; Smith, G. S. Appl. Catal. A Gen. 2011, 393, 231.

(c) Hertrich, M. F.; Scharnagl, F. K.; Pews-Davtyan, A.; Kreyenschulte, C. R.; Lund, H.; Bartling, S.; Jackstell, R.; Beller, M. Chem.-Eur. J. 2019, 25, 5534.

(d) Molnar, A. Coord. Chem. Rev. 2019, 388, 126.

(e) Antony, R.; Arun, T.; Manickam, S. T. D. Int. J. Biol. Macromol. 2019, 129, 615 .

[27] Makhubela, B. C. E.; Jardine, A.; Smith, G. S. Green Chem. 2012, 14,338 .

[28] (a) Shifrina, Z. B.; Matveeva, V. G.; Bronstein, L. M. Chem. Rev. 2020, 120, 1350.

(b) Kramer, S.; Bennedsen, N. R.; Kegnæs, S. ACS Catal. 2018, 8, 6961.

(c) Sun, Q.; Dai, Z.; Meng, X.; Xiao, F.-S. Chem. Soc. Rev. 2015, 44, 6018.

(d) Kaur, P.; Hupp, J. T.; Nguyen, S. B. T. ACS Catal. 2011, 1, 819.

[29] Sun, Q.; Jiang, M.; Shen, Z.; Jin, Y.; Pan, S.; Wang, L.; Meng, X.; Chen, W.; Ding, Y.; Li, J.; Xiao, F.-S. Chem. Commun. 2014, 50,
11844.

[30] Jiang, M.; Yan, L.; Ding, Y.; Sun, Q.; Liu, J.; Zhu, H.; Lin, R.; Xiao, F.; Jiang, Z.; Liu, J. J. Mol. Catal. A: Chem. 2015, 404, 211.

[31] Sun, Q.; Aguila, B.; Verma, G.; Liu, X.; Dai, Z.; Deng, F.; Meng, X.; Xiao, F.-S.; Ma, S. Chem 2016, 1, 628.

[32] Tang, Y.; Dong, K.; Wang, S.; Sun, Q.; Meng, X.; Xiao, F.-S. Mol. Catal. 2019, 474, 110408.

[33] Sun, Q.; Dai, Z.; Liu, X.; Sheng, N.; Deng, F.; Meng, X.; Xiao, F. S. J. Am. Chem. Soc. 2015, 137, 5204

[34] Li, C.; Sun, K.; Wang, W.; Yan, L.; Sun, X.; Wang, Y.; Xiong, K.; Zhan, Z.; Jiang, Z.; Ding, Y. J. Catal. 2017, 353, 123.

[35] Li, C.; Xiong, K.; Yan, L.; Jiang, M.; Song, X.; Wang, T.; Chen, X.; Zhan, Z.; Ding, Y. Catal. Sci. Technol. 2016, 6, 2143.

[36] Wang, Y.; Yan, L.; Li, C.; Jiang, M.; Wang, W.; Ding, Y. Appl. Catal. A Gen. 2018, 551, 98 .

[37] Wang, Y.; Yan, L.; Li, C.; Jiang, M.; Zhao, Z.; Hou, G.; Ding, Y. J. Catal. 2018, 368, 197

[38] Jia, X.; Liang, Z.; Chen, J.; Lv, J.; Zhang, K.; Gao, M.; Zong, L.; Xie, C. Org. Lett. 2019, 21, 2147.

[39] (a) Johnson, J. R.; Cuny, G. D.; Buchwald, S. L. Angew. Chem., Int. Ed. Engl. 1995, 34, 1760

(b) Agabekov, V.; Seiche, W.; Breit, B. Chem. Sci. 2013, 4, 2418.

(c) Fang, X.; Zhang, M.; Jackstell, R.; Beller, M. Angew. Chem., Int. Ed. 2013, 52, 4645 .

(d) Zhang, Z.; Wang, Q.; Chen, C.; Han, Z.; Dong, X.; Zhang, X. Org. Lett. 2016, 18, 3290.

[40] Liang, Z.; Chen, J.; Chen, X.; Zhang, K.; Lv, J.; Zhao, H.; Zhang, G.; Xie, C.; Zong, L.; Jia, X. Chem. Commun. 2019, 55, 13721.

[41] Dong, K.; Sun, Q.; Tang, Y.; Shan, C.; Aguila, B.; Wang, S.; Meng, X.; Ma, S.; Xiao, F.-S. Nat. Commun. 2019, 10, 3059.

[42] Wang, T.; Wang, W.; Lyu, Y.; Xiong, K.; Li, C.; Zhang, H.; Zhan, Z.; Jiang, Z.; Ding, Y. Chin. J. Catal. 2017, 38, 691 